\title{
Data Mining for Gene Mapping
}

\author{
Hannu Toivonen, Päivi Onkamo, Petteri Hintsanen, \\ Evimaria Terzi, and Petteri Sevon \\ Department of Computer Science and \\ Helsinki Institute of Information Technology \\ PO Box 26 (Teollisuuskatu 23) \\ FI-00014 University of Helsinki, Finland \\ E-mail: hannu.toivonen@cs.helsinki.fi,paivi.onkamo@cs.helsinki.fi, \\ petteri.hintsanen@cs.helsinki.fi, evimaria.terzi@cs.helsinki.fi, \\ petteri.sevon@cs.helsinki.fi
}

April 1, 2004

\begin{abstract}
Localization of disease susceptibility genes to certain areas in the human genome, or gene mapping, requires careful analysis of genetic marker data. Gene mapping is often carried out using a sample of individuals affected by the disease of interest and a sample of healthy controls. From a data mining perspective, gene mapping can then be cast as a pattern discovery and analysis task: which genetically motivated marker patterns help to separate affected individuals from healthy ones?

The marker data constitutes haplotypes: a haplotype is a string of genetic markers from one chromosome. Individuals who share a common ancestor, such as those that have inherited the disease gene from this individual, potentially share a substring in their haplotypes. Classification or association analysis of haplotypes is thus one approach to gene mapping. Further, analyzing the similarities of haplotypes and clustering them can provide insight to genetic relationships of individuals, to different mutations, and thus to the genetic etiology of the disease.

We describe and illustrate data mining approaches to gene mapping using haplotypes: association analysis, similarity analysis, and clustering. The association-based gene mapping methods have been found to perform well and are being routinely applied in gene mapping projects.
\end{abstract}

Keywords: gene mapping, association analysis, haplotype, similarity, clustering 
Modern biomedical research is uncovering the pathology of diseases once considered to be hopelessly complex and incurable. A great deal of this progress can be attributed to gene mapping, i.e., localization of disease susceptibility genes to certain areas in the human genome by a combination of state-of-the-art laboratory and computational methods.

Gene mapping is often based on analysing genetic sequences called haplotypes. (We will review basic concepts of genetics in more detail in the next section; here we aim to give a brief introduction to the topic of this chapter.) A haplotype is a sparse representation of DNA in (some part of) one chromosome: only the contents of some selected polymorphic locations of the chromosome are included as symbols in the haplotype. In any particular study, haplotypes are strings of a fixed length. When inherited from generation to generation, haplotypes are recombined by cross-overs. This adds variance to the observed haplotypes, and the variance reflects the history of each haplotype: two haplotypes that have a common ancestor potentially share a segment in common from that ancestor. In so-called association mapping, geneticists search for segments that are over-represented in patients of a given disease. The locations of those segments are likely sites of genes that affect the disease, as the segments are potentially inherited from a common ancestor together with the gene.

In this chapter we study data mining of haplotypes. A central goal is gene mapping, but we also consider haplotype similarity and clustering as tools to analyse haplotypes and find some structure in their relationships. All the methods we propose are based on discovering regularities or similarities in haplotypes; in the case of gene mapping this is done in relationship to the disease/healthy status of individuals in the study. A big challenge is the large amount of stochasticity in the data: the recombination process that has led to the observed haplotypes is stochastic, and the disease status typically correlates only weakly with any single gene.

Association mapping has several alternative formulations as a data mining problem (Section 3). The first is an extension of association rule mining: the task is to find sets or sequences of polymorphic locations and their variants ("attribute-value pairs") that are associated with the disease status with a high confidence. A straightforward application of association rules does not work, however. Three issues need to be addressed: specification of the pattern language, prediction of gene position based on discovered patterns, and removing the effect of random associations. An alternative formulation is classification: use a machine learning method to classify individuals to cases and controls, and predict a gene to be close to the polymorphic locations used by the classifier. 
Association mapping is based on the assumption that several carriers of the gene have inherited it from a common ancestor and therefore they share haplotype fragments. Similarity measures for individuals are a useful tool for assessing how closely related patients are and for finding structure in the haplotypes (Section 4). Clustering, based on similarity measures or directly on genetically motivated concepts, such as haplotype sharing, can be used to locate groups of individuals that are likely to share the same genetic etiology (Section 5).

The methods we provide are intended to be used as exploratory tools by geneticists. Like in any real data mining task, the user's expertize and insight are in a key role. They are needed in choosing the methods and parameter values, they are crucial in interpreting the results and in designing better ways of mining the data. We hope that these tools will help the geneticist to make useful discoveries.

In Sections 3-5 we will describe how different data mining approaches can be applied on gene mapping and closely related problems, and illustrate the methods using both synthetic and real data. Section 6 concludes with a discussion.

\section{Genetic concepts}

The human genome is organized into 23 different chromosomes, ${ }^{1}$ each present in every cell as two homologous copies (Figure $1 \mathrm{~A}$ ), one from mother and another from father. A chromosome is a single, giant DNA molecule, consisting of millions of consecutive pairs of nitrogenous bases, A-T (adenine and thymine), and C-G (cytosine and guanine), which form the well-known double helix structure with 4-letter alphabet. Most of the DNA has no known functional relevance; only minority of DNA is estimated to be genes or their regulatory factors (Figure $1 \mathrm{~B}$ ).

\section{(Figure 1 to be placed approximately here)}

The order of the bases and genes is the same from individual to individual, with only minimal variation: one of the most recent estimates, by Lon Cardon (in his presentation in the Annual Meeting of The American Society of Human Genetics, 2003) is that there are individual differences in 1 out of 330 base pairs. This variation inside the genome is utilized as genetic markers (Figure $1 \mathrm{C})$ : the alternative forms of the markers, alleles, can readily be distinguished from each other using standard laboratory methods (genotyping), and therefore they can be used in comparing individuals or populations, and in estimating co-occurrence of a disease with certain combination of marker alleles. A haplotype is a string of alleles in an individual's chromosome. Haplotypes

\footnotetext{
${ }^{1}$ All genetic concepts that are printed in italics when mentioned for the first time can be found in the glossary in Table 1.
} 
can be considered sparse, economic representations of chromosomes, whose focus and density is set by the location and the density of the marker map used in the study. For genome scans the marker map covers the whole genome or a full chromosome, for fine mapping studies the markers are more densely located in a candidate area for a disease susceptibility gene.

A very basic phenomenon in genetics is that of recombination: a pair of homologous chromosomes (represented by haplotypes for a gene mapping study) exchanges genetic material in the process of gamete production (Figure 2). As a result, a chromosome transmitted from a parent to an offspring is not an exact copy of either parental chromosomes, but a mosaic of them. Consequently, recombination ensures that between-individual variation is maintained in each generation. (An a large scale, such as those typically used in gene mapping, the probability of recombination is approximately constant along the chromosome, and the number of recombinations correlates well with the physical distance in the chromosome.) Recombination is the key factor for gene mapping: since it fragments haplotypes, the genealogies of different loci in the genome are different, and this helps to localize genes.

\section{(Figure 2 to be placed approximately here)}

In association mapping, correlation between the disease or trait (phenotype) and markers is sought in a sample of affected and healthy individuals from a given population (Figure $3 \mathrm{~A})$. It is assumed that disease mutations derive from one ancestral chromosome (thus, they are identical by descent, IBD), where a single mutation occurred a long time ago. (In contrast, alleles which are chemically identical but cannot be traced to common ancestor are identical by state, IBS.) As the generations have passed, the disease mutation has been transmitted onward, while recurrent recombinations have narrowed down the stretch of the ancestral haplotype around the mutation. Therefore, in the present generation, one observes a haplotype segment overrepresented in the affected individuals compared to the unaffected (Figure $3 \mathrm{C}$ ). There is then linkage disequilibrium (LD) between the actual disease gene and the surrounding markers. The longer the time which has passed since the original ancestral mutation, the shorter is the ancestral segment that contains the mutation.

\section{(Figure 3 to be placed approximately here)}

Association methods are utilized when (1) prior candidate genes exist, or (2) initial linkage to a genetic region has been observed. Compared to association mapping, linkage analysis, the search

of co-segregation of marker alleles and disease in carefully chosen pedigrees, is the means to get an initial clue of a position of disease genes (Figure 3 B). Even though linkage analysis is not very accurate, as only few recombinations can be expected to happen in a chromosome during a couple 
of generations, it has proven very useful in locating areas where fine-mapping efforts should be concentrated. Ultimately, both linkage analysis and association methods search for shared genetic factors between affected individuals, the difference lying in the size of the "pedigree" concerned (Figure 3). In this article, we will only consider association mapping.

We will describe novel data mining approaches to association mapping (and the term "gene mapping" in the rest of this chapter refers to association mapping, not linkage mapping). Due to historical reasons, the vast majority of gene mapping methodology is based on statistical modelling, in a field that is referred to as "genetic epidemiology". The idea of applying data mining methods to the mapping problem is quite unique, and we are not aware of any similar publications. We will illustrate our methods with two different data sets.

1. A simulated but realistic data set. The simulated population is an isolate whose age is 20 generations; the marker map is relatively sparse and covers a small chromosome. The final sample consists of 200 cases and 200 controls. The simulation procedure is described in more detail in Appendix A.

2. A data set for SLE (systemic lupus erythematosus), where a founder mutation haplotype has been found very recently [13]. SLE is a rare autoimmune disorder. Its pathogenesis is polygenic with potential environmental effects. Genes are not yet very well known, though some candidates have been found by genome scans. We concentrate on a linkage peak area in chromosome $14[13,11]$. Both the linkage peak, as well as an association peak were shown to reside at the same position, in close vicinity of marker D14S1055 at $50.30 \mathrm{cM}$, which for the present knowledge is, or is very near to, the actual position of the susceptibility gene. The proportion of affected individuals actually carrying the particular founder mutation in chr 14 is on the order of $20 \%$, which is typical for multifactorial diseases.

\section{(Table 1 to be placed approximately here)}

\section{Gene mapping}

\subsection{The problem}

The goal in gene mapping is to locate disease-predisposing genes. A usual setting consists of cases and controls: cases are persons that have the disease that is being studied, and controls are healthy individuals. The existence and importance of a genetic component in the etiology of the disease has usually already been identified, so the question now is about where the disease 
susceptibility (DS) gene or genes are located. Given the haplotypes of cases and controls, the basic idea in association mapping is to search for genetic patterns that are more common within cases than controls. Sometimes the trait to be analyzed is quantitative, e.g., blood pressure, rather than dichotomous case vs. control. In this chapter we consider case-control settings, and will only briefly outline how to extend the methods for quantitative traits and covariates.

In the following we assume that the gene has been initially mapped to an area on a chromosome. The area has been saturated with markers and genotyped in a number of individuals. Each individual in our sample contributes a chromosome pair (one maternal and one paternal chromosome), so the number of chromosomes in the data is twice the number of individuals. For simplicity, we consider the input data as consisting of a set of disease-associated haplotypes (from the cases) and a set of control haplotypes (from the controls). It is typical that many or most of the disease-associated haplotypes do not carry the actual predisposing mutation, and many control haplotypes do carry it.

The association-based gene mapping problem The input consists of a marker map $M=(1, \ldots, m)$, a set $A=\left\{A_{1}, \ldots, A_{p}\right\}$ of disease-associated haplotypes $A_{i}$ over map $M$, and a set $C=\left\{C_{1}, \ldots, C_{q}\right\}$ of control haplotypes $C_{j}$ over map $M$. A haplotype $H$ over map $M$ is a vector $H=\left(a_{1}, \ldots, a_{m}\right)$ of alleles, where $a_{i} \in \mathcal{A}_{i}$ and $\mathcal{A}_{i}$ is the set of alleles at marker $i$. The task is to predict the location of a disease susceptibility gene on the map $M$.

\subsection{Standard data mining formulations of the gene mapping problem}

The problem formulation is vague: it does not say anything about how to predict the gene location. We next briefly review possible straightforward data mining formulations of the problem.

From a data mining or machine learning view point, gene mapping can be seen as a classification problem. This seems obvious, since the input data is readily classified into cases and controls. The strategy for gene mapping as a classification task is as follows. First, learn to predict whether a haplotype is a case or a control. Then, by looking at the prediction model, identify the area of the chromosome that is most important for the classification. The gene is potentially in this area.

While the classification approach may seem tempting, due to the existence of a large variety of effective and well known classification methods, there are severe problems in any straightforward application of machine learning methods to this problem. First, the search space is usually huge with respect to the number of training instances. The number of haplotypes is typically in the order of tens or hundreds, while the number of markers can be of the same order of much larger. Machine learning methods would often find over-fit classifiers that perform perfectly with the 
available training data, but whose predictions are based on totally irrelevant markers. Further, the data is extremely noisy. Both cases and controls contain both mutation carrying and non-carrying haplotypes (class noise), and haplotypes have errors and missing data (attribute noise). Finally, depending on the classifier, it can be difficult to tell which markers are the most important for classification.

Another possible approach is to use association rules of the form $X \rightarrow C$, where $X$ is a set of (marker, allele) pairs and $C$ is the case/control status. This is similar to looking for conjunctive classification patterns, and this approach shares the problems of the classification approach. Although the pattern language is quite restricted and therefore the danger of over-fitting is smaller, association rules still typically consist of markers that are not related to the mutation. Still another possibility from machine learning and data mining would be to use feature selection methods to rank or choose the markers that are most important and thus potentially close to the gene. Again, in most cases, irrelevant markers would be identified as most important ones.

\subsection{Haplotype pattern mining}

Like practically any application of data mining or machine learning, the gene mapping problem requires careful engineering of the types of patterns to be used, i.e., feature construction. The following three issues need to be addressed:

1. definition of a pattern language that expresses meaningful concepts of the problem at hand,

2. prediction of gene locus based on discovered patterns, and

3. removing the effect of random associations.

We next describe Haplotype Pattern Mining (HPM), a method that has been successfully applied on gene mapping, and explain how it solves these three issues.

HPM is based on the simple observation that linkage disequilibrium with the DS gene is likely to be strongest around it and, consequently, the gene locus is likely to be where most of the strongest associations are. The method looks for haplotype patterns, and predicts the DS locus to be where strongly disease-associated haplotype patterns are. We give a declarative specification of the HPM method after Toivonen et al. [21]; implementation details can be found elsewhere $[21,22]$.

Haplotype patterns and disease association We examine linkage disequilibrium by looking for haplotype patterns that consist of a set of nearby markers, not necessarily consecutive ones. 
Given a marker map $M=(1, \ldots, m)$, a haplotype pattern $P$ on $M$ is a vector $\left(p_{1}, \ldots, p_{m}\right)$, where $p_{i} \in \mathcal{A}_{i} \cup\{*\}$ for all $i, 1 \leq i \leq m$, where $\mathcal{A}_{i}$ is the set of alleles at marker $i$, and * is the 'don't care' symbol. A haplotype pattern $P$ occurs in a given haplotype vector (chromosome) $H=\left(h_{1}, \ldots, h_{m}\right)$ if either $p_{i}=h_{i}$ or $p_{i}=*$ for all $i, 1 \leq i \leq m$.

Example 1 Consider a marker map of 10 markers. The vector

$$
P_{1}=(*, 2,5, *, 3, *, *, *, *, *)
$$

where $1,2,3, \ldots$ are marker alleles, is an example of a haplotype pattern. This pattern occurs, for instance, in a chromosome with haplotype $(4,2,5,1,3,2,6,4,5,3)$.

HPM is based on recognizing disease-associated haplotype patterns that are potentially identical by descent, i.e., derived from a common ancestor. Gaps are allowed in the patterns to better accommodate for mutations, errors, missing data, and recombinations.

Example 2 Assume that a continuous chromosomal region including markers 2-5 is inherited from a common founder by a number of individuals, and that a marker mutation early in the coalescence history of the disease chromosome has changed the allele in marker 4 for a large fraction of current chromosomes. The haplotype shared by these individuals can be expressed as a haplotype pattern of the form of $P_{1}$ in Example 1.

Example 3 Assume that a continuous chromosomal region including markers 2-5 is inherited from a common diseased founder by a number of individuals. Errors in genotyping marker 4 may lead to a situation where a continuous haplotype pattern is not as significantly associated to the disease status as the one with a gap.

Errors of another type can be introduced in the construction of marker maps, by inferring a wrong order of markers. Assuming the physical order of markers 4 and 5 is actually the reverse, situations may occur where pattern $P_{1}$ is observed for continuous shared regions.

Gaps caused by marker mutations and errors are short, whereas missing information can span several consecutive markers, depending on how the data has been collected. Long gaps could be introduced by double recombinations, but they are rare on the genetically short distances where patterns can be observed in the first place. Since long patterns are not likely to exist, at least not in significant amounts, it can be useful for performance reasons to restrict the length of the patterns that are used in gene localization. 
Assume a haplotype pattern $P=\left(p_{1}, \ldots, p_{m}\right)$. The (genetic) length of $P$ is the maximum genetic distance (in Morgans) between any two markers $i, j$ with $p_{i} \neq * \neq p_{j}$. Gaps are maximal subsequences of 'don't care' symbols, excluding the tails of the pattern: a gap in $P$ is a contiguous sequence $p_{u}, \ldots, p_{v}$ of alleles, where

1. $p_{i}=*$ for all $i, u \leq i \leq v$ (the gap consists of 'don't care' symbols),

2. $u>1$ and $v<m$ (the gap is not at either end of the pattern), and

3. $p_{u-1} \neq *$ and $p_{v+1} \neq *$ (the gap is bounded by alleles, rather than 'don't care' symbols). The length of the gap is $v-u+1$.

The HPM algorithm takes as parameters the maximum number and maximum length of gaps, as well as the maximum length of patterns to be considered.

We use the signed $\chi^{2}$ statistic, denoted $\pm \chi^{2}$, to measure the marker-disease association. A signed version of the measure is used in order to discriminate disease association from control association, i.e., from protective haplotypes. The signed $\chi^{2}$ measure $\pm \chi^{2}(P)$ of a haplotype pattern $P$ is the standard $\chi^{2}$ measure where the sign is positive if the relative frequency of $P$ is higher in cases than in controls, and negative otherwise. Given a positive association threshold $x$, we say that $P$ is strongly associated with the disease if $\pm \chi^{2}(P) \geq x$. Given the data - markers $M$, a set $A$ of disease-associated haplotypes and a set $C$ of control haplotypes on $M-$ and an association threshold $x$, we denote the collection of all strongly disease-associated patterns by $\mathcal{P}$, i.e., $\mathcal{P}=\left\{P\right.$ is a haplotype pattern on $\left.M \mid \pm \chi^{2}(P) \geq x\right\}$.

If pattern parameters are specified - a maximum genetic length, a maximum number of gaps, or a maximum length for gaps — the set $\mathcal{P}$ is refined by requiring that these additional restrictions are also fulfilled by the patterns in $\mathcal{P}$. Fisher's exact test could also be used instead of $\chi^{2}$, especially if any of the values used in the computation of $\chi^{2}$ are small. Since we do not use $\chi^{2}$ for exact $p$ value computations, the selection of the test is not critical.

Prediction of gene locus Haplotype patterns close to the DS locus are likely to have stronger association than haplotypes further away; consequently the locus is likely to be where most of the strongest associations are. The marker frequency $f(i)$ of marker $i$ (with respect to $M, A, C, x$ as above) is the number of strongly disease-associated patterns that contain marker $i$, possibly in a gap:

$$
f(i)=\mid\left\{P=\left(p_{1}, \ldots, p_{m}\right) \in \mathcal{P} \mid \text { there exist } t \leq i\right.
$$


and $u \geq i$ such that $\left.p_{t} \neq * \neq p_{u}\right\} \mid$.

The idea is that each haplotype pattern roughly corresponds to a continuous chromosomal region, potentially identical by descent, where gaps allow for corruption of marker data. While markers within gaps are not used in measuring the disease association of the pattern, the whole chromosomal region of the pattern is relevant under the assumption of the region being identical by descent.

The marker frequency gives a score for each marker. On the condition that we assume a DS gene to be present, e.g., based on linkage analysis, we would predict the gene to be somewhere close to the markers with largest frequencies. As a point prediction we can simply give the locus of the most frequent marker: the HPM point prediction of DS gene locus is the location of the marker $i$ that has maximal frequency $f(i)$.

This prediction method does not, of course, imply that we assume the DS locus to really overlap with the marker; we simply predict at the granularity of marker density. Consequently, the optimal point predictions of our method are within one half of the inter-marker distance from the true loci.

Removing the effect of random associations The frequency-based approach has some potentially severe problems: uneven marker spacing, different allele distributions of markers, unevenly distributed missing and erroneous data, and background linkage disequilibrium all can change the observed marker frequencies from what they would be in an ideal situation. This can lead to a situation where, for instance, a large number of patterns are observed in a certain region due to larger heterogeneity in and weaker LD between the markers. Some of these patterns can seem significant just by random. To avoid these problems we estimate the statistical significance of marker frequencies. Given a marker $i, p(i)$ is the statistical significance of the frequency $f(i)$ of $i$ under the null hypothesis that 'chromosomes are drawn from the same distribution', i.e., that there is no gene effect.

Marker significance can be estimated by standard permutation tests. Under the null hypothesis case and control haplotypes come from the same distribution; by randomly permuting the statuses of haplotypes we obtain samples from the null distribution. We generate for instance 10000 such random permutations and compute the marker frequencies in each of those. The $p$ value $p(i)$ is then estimated as the fraction of permutations that achieved frequency of at least $f(i)$.

The HPM significance-based point prediction is the location of the marker $i$ that has minimal (i.e., highest) significance $p(i)$. The use of marker significances can be illustrated as follows. Consider the frequency of a fixed marker as the test statistic. If it is very unlikely that at least that large a frequency occurs by chance, then it is likely that the DS locus is genetically close to that 
marker. The significance-based approach predicts the DS gene to be in the vicinity of the marker with the smallest $p$ value. Consecutive markers are dependent, and thus a large number of mutually dependent $p$ values are produced. This is not a problem, however, since we do not use the $p$ values for hypothesis testing, but only for ranking markers.

\subsection{HPM algorithm}

In a nutshell, the HPM algorithm is as follows (Figure 4). First, given an association threshold for the $\chi^{2}$ statistic, a lower bound can be derived for the frequency of strongly disease-associated haplotype patterns (Step 1). Second, given such a frequency threshold, all patterns exceeding the threshold can be enumerated efficiently with a fairly straightforward depth-first search method (Step 2; see [22] for more information about these first two steps).

\section{(Figure 4 to be placed approximately here)}

The strengths of the patterns are then computed, and the the strong ones are used to compute the marker frequencies $f(i)$ (Steps 3-4). The permutation testing is carried out next (Steps 5-9). The same set of frequent patterns is reused in every iteration, but the strengths are recomputed based on the randomized status fields. The final output is a sorted list of markers and their significances. The first markers in the output have the highest likelihood of being close to the DS gene.

\subsection{Extensions of HPM}

It is relatively easy to modify HPM to accommodate different kinds of input data. QHPM [20, 16] is an extension of HPM which can handle quantitative traits and covariates, such as body mass index, smoking habits, etc. The pattern-trait association is measured via a linear model having the trait as the response variable and the covariates and an indicator variable for the occurrence of the pattern as explanatory variables. The significance of the pattern as an explanatory variable can be tested by comparing the best fit model to the best fit model where the coefficient corresponding to the pattern is zero. We also replaced marker frequencies with a score function measuring the skew of the $p$ values of the overlapping patterns.

F-HPM by Zhang and Zhao [23] uses family data instead of independent haplotypes. A familybased association test proposed by the same authors is used for measuring the pattern-trait association. 


\subsection{Examples}

Figure 5 gives graphical representations of the output of HPM on the simulated data set (Appendix A). The parameters for HPM were set as follows: $\chi^{2}$ threshold was 9 , maximum pattern length was 7 markers, maximum number of gaps was 1, and maximum length of gap was 1 marker. Permutation tests were carried out with 100,000 iterations. Negated logarithms of $p$ values are used to illustrate the significance-based predictions, as they are more intuitive than plain $p$ values: higher values mean stronger association and differences between small $p$ values are more easily observed. The signal is very strong for the locus on the right, and the number of permutations was not sufficient to differentiate the markers around the locus. With real data one usually does not expect to find such a strong signal. The locus on the left is more difficult to detect, and the peak is off the correct location by $5 \mathrm{cM}$.

\section{(Figure 5 to be placed approximately here)}

Next, we demonstrate HPM on real Type 1 diabetes data $[4,21]$ and the SLE data set described in Section 2. The original diabetes data consisted of affected 385 sib-pair families. There were 25 markers spanning over a $14 \mathrm{Mb}$ region. There are two known genes affecting risk for diabetes, very close to each other. The genes lie inside the HLA-complex, a region of very high LD, making the mapping task more difficult. We down-sampled the data to 200 disease-associated and 200 control haplotypes. The same parameters were used as with the simulated data set above. The result in Figure 6A indicates that HPM is capable of localizing genes even in presence of very strong background LD.

The SLE data set consisted of 104 disease-associated and 100 control haplotypes. There were 32 markers spread over a $25 \mathrm{cM}$ region. The results (Figure 6B) show suggestive association at $45-50 \mathrm{cM}$, coinciding with earlier linkage results [13]. Some of the patterns strongly associated with SLE are listed in Table 2.

(Figure 6 to be placed approximately here)

(Table 2 to be placed approximately here)

HPM has been found to be a valuable tool for narrowing down the region resulting from an initial linkage analysis. It has been utilized in several disease projects, for instance, asthma and high IgE [10, 19], SLE [12, 13] glioma [17], and dyslexia (unpublished). 


\section{Haplotype similarity}

Gene mapping, as described above, is indirectly based on finding similar haplotypes among the affected individuals. In this section we consider explicit similarity measures for haplotypes. Formally, given a set $G$ containing $n$ haplotypes with $m$ markers, we want to define a similarity function $\operatorname{sim}: G \times G \rightarrow[0,1]$, where 0 means total dissimilarity and 1 total similarity. This function allows us to quantitatively measure a (genetic) similarity between any pair of haplotypes taken from the set $G$. The idea is that the similarity is greater if the haplotypes are closely related and share more of the genome IBD (identical by descent). Depending on the genealogical properties of the haplotypes $G$ and the exact formulation of $\operatorname{sim}$, this function can be used e.g. to distinguish between different disease gene mutation carriers or to measure a relationship between haplotypes or individuals.

\subsection{Similarity measures}

Given a haplotype pair $H_{1}, H_{2} \in G$, we compare the alleles at the same locus (marker) within the haplotypes. By performing this pairwise comparison at every marker, we obtain a similarity vector $\vec{s}_{H_{1}, H_{2}}=\left(s_{1}, \ldots, s_{m}\right)$, where each element $s_{i}, 1 \leq i \leq m$, is a result of an allele comparison at the $i$ th marker: $s_{i}=1$ if the alleles at $i$ th marker match and $s_{i}=0$ otherwise. The similarity vector $\vec{s}_{H_{1}, H_{2}}$ is the base for all our similarity functions.

To begin with, we could simply count the number of $1 \mathrm{~s}$ in the vector and divide by $m$, which yields a similarity function $\operatorname{sim}\left(H_{1}, H_{2}\right)=\left(\sum_{i=1}^{m} s_{i}\right) / m$. Observe that $1-\operatorname{sim}\left(H_{1}, H_{2}\right)$ is a (normalized) Hamming distance between $H_{1}$ and $H_{2}$. The Hamming distance gives an equal weight to every match and completely ignores LD (linkage disequilibrium). Therefore it weakly distinguishes IBD sharing from IBS sharing: it is possible that most of the matching markers are identical by state and do not reflect true genetic relatedness. (Obviously, the probability that two alleles are identical by state is higher when the number of different alleles in the corresponding marker is low. SNP markers only have two alleles, so the probability of IBS sharing is considerably high.) Longer sequences of matching markers are more likely to be identical by descent due to the LD between adjacent markers. We consider two simple methods that give more weight for probable IBD sharing, and one a bit more elaborate method.

Elementary measures First we consider a sliding window technique. Fix a window width $w \in$ $\mathbb{N}$. For each marker $k$ in $\vec{s}_{H_{1}, H_{2}}$, we count the amount $a_{k}$ of matching markers covered by the win- 
dow starting at the marker: $a_{k}=\sum_{i=k}^{k+w-1} s_{i}$, where $s_{i}=0$ for $i \notin\{1, \ldots, m\}$ (we allow the window to slide "over the edges" of $\vec{s}_{H_{1}, H_{2}}$ ). The windowing technique is not sensitive to mismatches in the middle of the sequence of matches in $\vec{s}_{H_{1}, H_{2}}$. This property makes it robust against genotyping errors, missing data and point mutations, and gives a smooth weighting for consecutive or near-consecutive matches.

Now, compute the similarity as $a=\sum_{k=(-w+2)}^{m} a_{k}^{\alpha}$ for some constant $\alpha \geq 1$. The exponent $\alpha$ gives the desired weight for the possible observed LD: larger values of $\alpha$ put more emphasis on longer matches. Finally we normalize $a$ by dividing it by the maximum possible score $C=$ $(m-w+1) w^{\alpha}+2 \sum_{k=1}^{w-1} k^{\alpha}$ and let $\operatorname{sim}\left(H_{1}, H_{2}\right)=a / C$ be the normalized value.

In the second method, we consider each sequence of consecutive matches separately. Let $s_{i}, \ldots, s_{k}$, for $1 \leq i<k \leq m$, be a substring of $\vec{s}_{H_{1}, H_{2}}$ such that $s_{j}=1$ for all $i \leq j \leq k$ and $s_{i-1}=$ $s_{k+1}=0$. Denote by $S$ the set of all such sequences. Let $a=\sum_{s \in S}|s|^{\alpha}$ for some $\alpha \geq 1$, where $|s|$ is the length of the sequence $s$. Finally we normalize $a$ so that $\operatorname{sim}\left(H_{1}, H_{2}\right)=a / m^{\alpha}$.

As with the sliding window, constant $\alpha$ gives the desired weight for the possible observed LD. However, unlike the sliding window, this method does not allow any gaps in sequences of consecutive matches.

Second-order similarity In the second-order similarity (similar in spirit to external similarity [6]) we do not compare a pair of haplotypes directly to each other, but instead consider their relations with other haplotypes. The general idea can be described as follows: if two persons share a lot of (close) relatives, then they probably are (close) relatives, too. We use the above described elementary similarity measures to estimate which other haplotypes are related and to which degree with the two haplotypes at hand.

This second-order similarity is useful since the elementary similarity has a large variance due to the stochasticity of the recombination process and random sharing of alleles. The second order similarity helps us see more systematic relations between haplotypes. As an extreme example, suppose that two haplotypes $A$ and $B$ share a DS gene. Assume further that there have been recombinations very close to the gene in the genealogies of both $A$ and $B$, but on different sides of the gene. The haplotypes thus share only few alleles, if any, around the gene locus. The elementary measures obviously would not consider these haplotypes similar (unless $A$ and $B$ share a significant amount of alleles somewhere else by chance). If there are other haplotypes carrying the same mutation, they can help us see $A$ and $B$ as similar. Consider such a mutation carrier $C$ with its own history of recombinations, all further away from the gene. Then $C$ shares a haplotype 
fragment with $A$ (on one side of the gene), another fragment with $B$ (on the other side of the gene), and has high elementary similarity with both $A$ and $B$. With several other such haplotypes, we say that $A$ and $B$ are similar in the second order since they share many closely related haplotypes.

We use the observed correlation of elementary similarities to measure the second-order similarity. First we construct a similarity matrix SM, where $\mathrm{SM}_{H_{1}, H_{2}}=\operatorname{sim}\left(H_{1}, H_{2}\right)$ for $H_{1}, H_{2} \in G$ (here we use the haplotype set $G$ also as an index set), by applying either of the elementary methods described in the previous section. Denote by $\operatorname{sm}_{H_{1}}$ and $\mathrm{sm}_{H_{2}}$ the $H_{1}$ th and $H_{2}$ th row in the matrix SM with the $H_{1}$ th and $H_{2}$ th columns removed (in other words, we drop $H_{1}$ and $H_{2}$ from inspection). We define the second-order similarity between two haplotypes $H_{1}, H_{2} \in G$ as the correlation coefficient between these rows:

$$
\mathrm{SM}_{H_{1}, H_{2}}^{2}=\frac{\operatorname{Cov}\left(\mathrm{sm}_{H_{1}}, \mathrm{sm}_{H_{2}}\right)}{\sqrt{\operatorname{Var}\left(\mathrm{sm}_{H_{1}}\right) \cdot \operatorname{Var}\left(\mathrm{sm}_{H_{2}}\right)}}
$$

where $\mathrm{SM}^{2}$ is the second-order similarity matrix and $\mathrm{SM}_{H_{1}, H_{2}}^{2} \in[-1,1]$.

Comments on the similarity measures Although the similarity methods discussed above are rather simple, they seem to perform quite well in practice. The simplicity has also advantages: we can easily convert a similarity function into distance function by applying some strictly decreasing transformation. For example, consider a mapping $d: G \times G \rightarrow[0,1], d\left(H_{1}, H_{2}\right)=1-\operatorname{sim}\left(H_{1}, H_{2}\right)$, where $\operatorname{sim}\left(H_{1}, H_{2}\right)$ is one of the elementary methods described above. It can be proved that $d$ satisfies metric properties, which is important in some applications. Both methods can be trivially implemented into algorithms with $\Theta(m)$ time complexity.

However, defining the constants $w$ and $\alpha$ is not straightforward. They should be large enough to adequately distinguish between probable IBD sharing and random sharing. Some proposed models (see e.g. [14]) for estimating the expected length of an IBD-shared segment, given an estimate of the age of the population, could be helpful here. On the other hand, the parameters should not be too large: this can lead to a situation where similarity values are negligible and, hence, the methods separate haplotypes inadequately. To be on a safe side, we suggest to start the exploration with relatively small constants $(w \leq 5, \alpha \leq 2)$, especially with long marker maps.

\subsection{Identification of most likely mutation carriers}

To illustrate how the proposed similarity measures can be applied, we consider the problem of identifying most likely mutation carrying chromosomes. The task is far from trivial. First, each individual contributes two chromosomes and carries the mutation potentially in one of them, both, or none. Second, a typical study setting in disease gene mapping is one with a so called complex 
disease, where several genes and environmental factors contribute to the disease susceptibility, and potentially only a small proportion of the chromosomes carry any particular disease mutation. A single haplotype can carry several mutations, although we restrict our attention to only single mutation per haplotype for simplicity.

Since carriers sharing a common mutation potentially share several markers IBD around the mutation locus (depending on the time since the last common ancestor and the density of the marker map), they are likely to be similar to each other. In addition, any two persons in the dataset could be related via a common ancestor, so mutation sharing is not the only source of similarity. Finally, haplotypes can be similar just by chance. We show how the proposed similarity measures can be applied to distinguish between carrier and non-carrier haplotypes.

Separating non-carriers from carriers Non-carrier haplotypes are not likely to share substantial amounts of markers with many other haplotypes, since there should be no systematic relationship between them. Therefore we can expect that the non-carrier haplotypes do not have many close neighbors. Formally, we classify haplotype $H \in G$ as non-carrier if $q(H)<t$ for some qualifying function $q: G \rightarrow \mathbb{R}$ and threshold constant $t \in \mathbb{R}$. Otherwise we classify $H$ as carrier. For the qualifying function $q$ we set $q(H)=|\bar{B}(H, \varepsilon)|$, where $\bar{B}(H, \varepsilon)=\left\{H_{2} \in G: \operatorname{sim}\left(H, H_{2}\right) \geq \varepsilon, H \neq H_{2}\right\}$ denotes the (closed) $\varepsilon$-neighborhood of the haplotype $H$. Parameter $\varepsilon$ is the amount of similarity we require for a given pair of haplotypes to be considered as close neighbors. By adjusting the threshold parameter $t$ we can control the number of remaining haplotypes. This model introduces two new parameters with which the geneticist can experiment in exploratory haplotype analysis. Usually we would begin with larger values and gradually descend down if the amount of noncarriers seems to be implausible.

The model for separating carriers and non-carriers is simple and guarantees only that the predicted carrier haplotypes have a certain number of close neighbors. It does not require any relation between the carriers. If we are very unlucky, we just get a set of predicted carriers which are dissimilar to each other. We could try to construct the carrier set $S \subset G$ more elaborately, though. We want the set $S$ to contain dense subsets $S_{1} \subset S, \ldots, S_{k} \subset S$, such that the haplotypes in a certain subset $S_{i}$ consist of similar haplotypes. This, obviously, is a clustering problem. We will return to clustering in the next section.

We construct a set $S \subseteq G$ of most likely mutation carriers by iteratively removing least related (non-carrier) haplotypes from the haplotype set $G$ with the qualifying function $q$ defined in the previous section. First we compute similarities between all haplotype pairs in the set $G$. Then we 
discard all haplotypes $H \in G$ for which $q(H)<t$ with some fairly large threshold and neighborhood "radius" (for example $t=10$ and $\varepsilon=0.3$ using second-order similarity). If all of the haplotypes get discarded, we drop the threshold $t$ by one and prune again. If the threshold drops to one (or some other lower bound we set for the density), we have to adjust $\varepsilon$ and try again. We emphasize the exploratory nature of the process, and the use of genetic insight in the interpretation of the results.

Every haplotype in the set $S$ has a certain number of close neighbors. However, these haplotypes do not need to be close to each other. Therefore we repeat the pruning described above for the set $S$ without adjusting the parameters $t$ and $\varepsilon$. This removes possible "distant" haplotypes from the set $S$. Denote the resulting set of this iteration by $S^{\prime}$. If $|S|=\left|S^{\prime}\right|$, we are done (now the set $S$ contains only haplotypes which form group or groups). Otherwise we set $S=S^{\prime}$ and repeat the pruning again. Since $|S|$ decreases monotonically, iterating will stop. The subset $S$ might end up empty, in which case we have to relax threshold parameters.

\subsection{Examples}

In our first set of examples we use the simulated dataset with 400 haplotypes from affected individuals with 101 microsatellite markers (Appendix A). There are three different mutations (labeled M2, M1 and M11) in the sample represented by 58, 56 and 42 carriers. (Two other mutations still present in the final population are ignored, because they have only one carrier haplotype each.) Thus, we have four classes, one for each mutation and one for non-carriers.

Similarity between classes First we verify that carriers of a particular mutation are indeed similar to each other. This is done by calculating the median similarity with respect to all classes for each haplotype. We can do this since we know whether a certain haplotype in the simulated dataset is a carrier or not, and if it is, which mutation the haplotype carries (that is, we know the correct class for each haplotype). More precisely, for each haplotype $H \in G$ and class $i$ we calculate

$$
S_{H_{i}}=\underset{\substack{H_{2} \in C_{i} \\ H_{2} \neq H}}{\operatorname{median}} \operatorname{sim}\left(H, H_{2}\right), \quad 1 \leq i \leq 4,
$$

where $C_{1}, \ldots, C_{4}$ are the known classes.

Figure 7 shows the distributions of $S_{H_{i}}$. Most of the carrier haplotypes have, as expected, greater median similarity within their own classes. Observe that most of the non-carrier haplotypes do not appear to be significantly similar to any group. This is a crucial property when predicting the carrier haplotypes.

(Figure 7 to be placed approximately here) 
Identification of mutation carriers We next apply the approximation heuristic to the prediction of mutation carriers. For the threshold parameters we set $\varepsilon=0.35$ and $t=5$ and use the second-order similarity with the sliding window method for measuring pairwise similarities between haplotypes (with parameters $w=5$ and $\alpha=2$ ). Our results are summarized in Table 3.

\section{(Table 3 to be placed approximately here)}

From the table we observe that all classes are present in the set of predicted carriers. Figure 8 illustrates the trade-off in selectivity. In the figure, two different parameter combinations have been used and the resulting sets are plotted onto the plane after multidimensional scaling. Although the heuristic discards a substantial amount of carriers during the process, the remaining haplotypes are the most likely carriers, and they are likely to show the most distinctive haplotype patterns of carrier haplotypes. They can be useful, for instance, for developing gene tests before the actual gene is recognized and can be tested directly.

\section{(Figure 8 to be placed approximately here)}

Structure discovery from real data In our last example we look for similarities in the real SLE dataset described in Section 2. There are 204 haplotypes with 32 microsatellite markers. Approximately 8.5 percent of the alleles are missing. When comparing two alleles, the result is conservatively considered to be a mismatch if either one of the alleles is missing.

We calculate the pairwise similarities with the second-order similarity using the sliding window method with parameters $w=5$ and $\alpha=2$. Figure 9A shows the whole dataset plotted onto a plane with Sammon's mapping. There are roughly two clusters, which do not seem to be in direct relation with the disease status or mutation.

To better distinguish the two clusters, we apply the approximation heuristic with parameters $\varepsilon=0.375$ and $t=10$. This removes exactly half of the haplotypes. We calculate the pairwise similarities for the remaining haplotypes with the second order similarity using the same parameters as before (Figure 9B). The two clusters are now more clearly visible. Again, the disease status or gene does not seem to have any significance in the separation of the two clusters. The two clusters suggest, however, that there is some clear structure in the data besides the disease status.

(Figure 9 to be placed approximately here) 


\section{Haplotype clustering}

\subsection{The clustering problem}

By haplotype clustering we aim to identify groups of related haplotypes. In some gene mapping studies, such groups could correspond to different disease gene mutations. Assume a population isolate created by a relatively small number of founders, constituting the initial generation. Further, assume that disease susceptibility mutations for the disease of interest have been introduced by few of the founders. Can the corresponding groups of carriers of different mutations be identified in the current population? This can be seen as a clustering problem: the goal is to group together individuals of the present population that have inherited specific regions of their haplotypes from specific individual founders. Solving this problem can be a rather difficult task, particularly when the number of generations between the initial and the final population is large, due to the large number of recombinations and mutations that might have occurred at multiple points.

The problem is closely related to gene mapping, as described in Section 3 . The main difference is that here we are not (directly) concerned with the disease status of the individuals and the goal is not to separate the control haplotypes from cases but to find groups of haplotypes that have inherited the mutation from the same ancestral haplotype.

Clustering is the process of grouping together items that have something in common. Several clustering algorithms exist, most of them requiring a measure of similarity between the items. (An extensive review of these clustering methods is provided for instance by Jaine et al. [2].) As discussed in previous sections, a consequence of the recombination process is that haplotypes that have inherited the mutation from the same ancestor are expected to share some more genetic material around the mutation locus, and haplotypes that share alleles in consecutive markers should be considered similar to each other.

This notion of similarity implies that conventional similarity measures are not very useful for haplotype clustering. Consider for example the three haplotypes shown in Table 4. The table shows the alleles of 3 haplotypes for markers 1 to 9 . By observation the substring 34 3 shared by $H_{1}$ and $H_{2}$ seems to be genetically significant, since it may correspond to a part of their common ancestral haplotype. However, using Hamming or Euclidean distance haplotype $H_{1}$ is closer to $H_{2}$ : Hamming_Distance $\left(H_{1}, H_{2}\right)=6$, Hamming_Distance $\left(H_{1}, H_{3}\right)=4$, and Euclidean_Distance $\left(H_{1}, H_{2}\right)=36$, Euclidean_Distance $\left(H_{1}, H_{3}\right)=5$. Since our goal is to identify groups of haplotypes that share a mutation from a common ancestor, the clustering method should be based on some groupwise similarity measure rather than any pairwise similarity. 
(Table 4 to be placed approximately here)

\subsection{Conceptual clustering}

Conceptual clustering builds on the idea that the clustering algorithm should produce clusters that can be described in a given language $\mathcal{L}$, which has been designed to express meaningful concepts of the domain. (For a discussion of the properties of certain conceptual clustering models, see [18].) In the sequel we describe the concept language as well as the haplotype clustering algorithms that have been employed for automating the concept formulation.

The concept language Given that the haplotypes of each cluster are expected to be genetically related by sharing a gene inherited from a common ancestor, the concept language should consist of expressions that describe shared haplotype segments. Due to recombinations, however, it is possible that there is no single haplotype segment that would both match most haplotypes in the (desired) cluster as well as separate them from other haplotypes. Therefore, the clusters are described by disjunctions of partially overlapping haplotype segments; the goal is that they are likely to be parts of ancestral haplotypes shared by subgroups. Disjunctions allow flexibility that is necessary due to different recombination histories of different haplotypes, while the overlap potentially contains the disease susceptibility gene, and also makes it more likely that the haplotypes within a cluster are related.

Example 4 An example of a disjunctive concept is shown in Table 5. Marker 88 is probably inherited from a common ancestor, while the different haplotype segments to the left and to the right are the results of different recombinations.

\section{(Table 5 to be placed approximately here)}

Formally, the haplotype cluster description language $\mathcal{L}$ consists of disjunctions of overlapping haplotype segments of the form

$$
i: a_{i}, a_{i+1}, \ldots, a_{i+L_{i}} \text { or } j: a_{j}, a_{j+1}, \ldots, a_{j+L_{j}} \text { or } \cdots k: a_{k}, a_{k+1}, \ldots, a_{k+L_{k}}
$$

where $i, j, \ldots, k$ are markers, $a_{i}$ is an allele at marker $i, L_{i}, L_{j}, \ldots, L_{k}$ are the lengths of the disjuncts, and there is at least one shared marker $h$ such that $i \leq h \leq i+L_{i}, j \leq h \leq j+L_{j}, \ldots, k \leq h \leq k+L_{k}$.

The clustering algorithm As potential disjuncts in the concepts, we consider all haplotype segments shared by more than a certain number of haplotypes. For finding them efficiently we use a 
slight modification of the Apriori algorithm [1]. We then focus on each marker in turn and construct the subsegment containment lattice of overlapping segments that contain this marker. An abstract description of the haplotype clustering algorithm is given in Figure 10, while some details of the method are discussed in the sequel.

\section{(Figure 10 to be placed approximately here)}

At each marker $i$ we construct the containment lattice $L A T T_{i}$ of the frequent haplotype segments that contain $i$ (Steps 1 and 2). In the first level of $L A T T_{i}$ we consider the alleles that are frequent in this marker and use them as the roots of the lattice. In the next level segments of length two that contain the marker-allele pairs already considered in the previous level are considered, and so on.

The use of the lattice structure biologically motivated. Assuming that a locus is identical by descent in the given haplotypes then the genealogy of the locus is tree-shaped. Under the assumption of no marker mutations this tree is contained in the haplotype segment lattice build at that locus. These assumptions are not fully realistic: first, only an unknown subset of haplotypes shares a mutation of interest IBD; second, marker mutations, errors and missing data can violate the second assumption. Since we do not know the loci of interest, we build a lattice at every marker locus to obtain a representative collection of lattices that potentially contain an interesting genealogy. Unlike the unknown trees, the lattices are unique and efficient to construct.

We next select the most promising lattices, based on the number of nodes in each lattice. Close to the mutation locus, where several haplotypes have segments identical by descend, the lattices are expected to have more internal nodes when compared to the number of internal nodes in the lattices for loci where sharing is by state only.

Finally we select the most descriptive nodes of each lattice and output their disjunction as a cluster description (Steps 4-6). We assign a score for each node (haplotype segment) of the lattice, based on the heuristic that a haplotype segment constitutes a good description if it is long and frequent. These two requirements of a good description are combined in the following heuristic, recursive definition of the score of a node $n$ :

$$
\operatorname{score}(n)=\left\{\begin{array}{cl}
\text { n.length }+ \text { n.frequency, } & \text { if } n \text { is a leaf } \\
\sum_{u \in \text { children_of }(n) \frac{\text { u.frequency }}{\text { n.frequency }} \times \operatorname{score}(u)} & \text { if } n \text { is not a leaf. }
\end{array}\right.
$$

For each lattice $L A T T_{j} \in S \_L A T T$ the score of the nodes in the lattice is evaluated and the nodes are sorted in decreasing order. A disjunction of the $p$ highest scoring haplotype segments is used as the cluster description. There can be logically redundant disjuncts in a cluster description; we have decided to keep them since they may be informative to the user, even if they do not affect cluster memberships. 


\subsection{Examples}

Figure 11 shows the distributions of different mutations (founder/locus pair) in clusters discovered in the simulated dataset. Most original mutations have disappeared in the course of the history of the population, and only three are left in the final sample $(1,2,11)$. Label NC in the graphs corresponds to non-carriers. Mutation 1 is best picked out by clusters 8-10, mutation 2 by cluster 7, mutation 11 by clusters 4 and 5, and non-carriers by none of the clusters, as expected. For our goal, best clusters have one dominant mutation. Clusters 4 and 7, that are among the best in this respect, are also those that correspond to the marker positions closest to the mutation loci. There is considerable noise in the results, i.e., clusters do not match to mutations one-to-one, but this is inherent due to the stochasticity of the data.

\section{(Figure 11 to be placed approximately here)}

The real SLE dataset (Section 2) has been used for clustering experiments as well. We carried out two experiments, where the goal was to test if we can re-discover some already known structure from this real data. In the first experiment we only considered affected individuals of the population (Table 6). There are 104 affected haplotypes, and 11 of them are actual mutation carriers. In the clusterings, done without information about who is a carrier and who is not, 10 out of 11 mutation carriers are in cluster 6 , together with 34 non-carriers. In general, the two classes are not separated well. An obvious reason is that the discovered cluster descriptions are so short that they will match a large number haplotypes. However, it is interesting to note that the topmost clusters contain more than half of the 11 carriers, i.e., the clusters tend to have been created around them rather than the dominating set of 93 predicted non-carriers.

In the second experiment we considered the whole population. In this case the dataset consists of the 104 affected individuals, 11 of which carry the mutation, and 100 control haplotypes, and all information about these classes was hold back in the clustering process. The resulting clusters (not shown) typically contain the same amount of cases and controls, and zero or one mutation carrier. It seems that in this case the signal from 11 carriers is too weak, and the clusters reflect haplotype patterns of the general population.

(Table 6 to be placed approximately here)

\section{Discussion}

Analysis of haplotypes is important for human health care. Gene mapping helps to locate disease susceptibility genes, similarity and clustering analysis in turn can be used to find different subtypes 
of the mutations or to develop diagnostic tests: a new patient probably carries a disease mutation if the haplotype is similar to many carrier haplotypes or falls into one of the carrier clusters.

In this chapter we described novel data mining approaches to haplotype analysis tasks. Practically none of the previous research on gene mapping has originated from computer science community, as the problem has been approached mostly with statistical perspectives. Data mining and machine learning can contribute to the field with their concepts and algorithms, as we have illustrated with associations and classification, similarity, and clustering. The association-based gene mapping methodology, HPM, has been applied successfully to real gene mapping studies and has been competitive with previous state of the art methods $[21,10,16,17,13]$. The similarity and clustering research is more recent and has yet to demonstrate its value in practical gene mapping.

The presented methods are mostly meant to be used as tools for exploratory data analysis. For instance, choosing parameter values for similarity computation is not straightforward. Suitable values depend on the dataset and application, and need to be tuned manually. Tests with simulated data sets are also one possible approach to finding roughly suitable values.

This chapter has two major lessons for data miners. First, gene mapping has a number of important problems where data mining can have interesting applications. Second, it is often crucial to tailor data mining methods to the problem at hand. Even though the data mining techniques in this chapter are quite simple, they are successful because they have been tuned for these particular problems: the similarity measures are novel, haplotypes are clustered using a suitable concept language, and an appropriate pattern language is also needed for finding associations that are useful for gene mapping.

Several interesting gene mapping related data analysis problems have not been covered here. First, most interesting hereditary diseases are affected by several genes, there are interactions between genes, and between genes and the environment. The signal to detect only one gene at a time might be weak, and models for multiple genes and interactions could be more powerful for gene mapping. Second, there are a number of recent directions for research on haplotypes. It has been observed that haplotypes are made up of blocks, within which recombinations are unlikely (see e.g. [5, 8]). Haplotype blocks thus tend to be inherited as whole from generation to generation. Identification and utilization of such blocks is now a popular topic. A closely related topic is marker selection (see e.g. [3]): given that haplotyping is expensive and not all markers can be used in a particular study, which markers are the most informative? Finally, haplotypes are not obtained directly from the wet lab. Instead, they need to be inferred from genotype data where for each marker there is a pair of alleles from the two chromosomes of the individual, without information 
about which allele belongs to the chromosome (haplotype) inherited from the mother, and which to the one from the father. Haplotyping is an interesting combinatorial problem (see e.g. [9, 7]). An alternative approach would be to try to map genes directly from the genotype data instead of using haplotypes.

\section{References}

[1] R. Agrawal, H. Mannila, R. Srikant, H. Toivonen, and I. Verkamo. Fast discovery of association rules. In Advances in Knowledge Discovery and Data Mining, pages 307-328 (1996).

[2] A. Jain, M. Murty, and P. Flynn. Data clustering: A review. ACM Computer Surveys, 31:264323 (1999).

[3] H. Avi-Itzhak, X. Su, and F. De La Vega. Selection of minimum subsets of single nucleotide polymorphisms to capture haplotype block diversity. In Pacific Symposium on Biocomputing, pages 466-477 (2003).

[4] S. Bain, J. Todd, and A. Barnett. The British Diabetic Association - Warren repository. Autoimmunity, 7:83-85 (1990).

[5] M. Daly, J. Rioux, S. Schaffner, T. Hudson, and E. Lander. High-resolution haplotype structure in the human genome. Nature Genetics, 29:229-232 (2001).

[6] G. Das, H. Mannila, and P. Ronkainen. Similarity of attributes by external probes. In Proceedings of the Fourth International Conference on Knowledge Discovery and Data Mining, pages 23-29 (1998).

[7] L. Eronen, F. Geerts, and H. Toivonen. A Markov chain approach to reconstruction of long haplotypes. In Pacific Symposium on Biocomputing 2004, pages 104-115 (2004).

[8] S. Gabriel, S. Schaffner, H. Nguyen, J. Moore, J. Roy, B. Blumenstiel, J. Higgins, M. DeFelice, A. Lochner, M. Faggart, S. Liu-Cordero, C. Rotimi, A. Adeyemo, R. Cooper, R. Ward, E. Lander, M. Daly, and D. Altshuler. The structure of haplotype blocks in the human genome. Science, 296:2225-2229 (2002).

[9] D. Gusfield. Inference of haplotypes from samples of diploid populations: Complexity and algorithms. Computational Biology, 8:305-324 (2001). 
[10] P. Kauppi, K. Lindblad-Toh, P. Sevon, H. Toivonen, J. Rioux, A. Villapakka, L. Laitinen, T. Hudson, J. Kere, and T. Laitinen. A second-generation association study of the 5q31 cytokine gene cluster and interleukin-4 receptor in asthma. Genomics, 77:35-42 (2001).

[11] S. Koskenmies, P. Lahermo, H. Julkunen, V. Ollikainen, J. Kere, and E. Widén. Linkage mapping of systemic lupus erythematosus (SLE) in Finnish multiplex families. Journal of Medical Genetics, 41:e2-5 (2004).

[12] S. Koskenmies, E. Widén, J. Kere, and H. Julkunen. Familial systemic lupus erythematosus in Finland. Journal of Rheumatology, 28(4):758-760 (2001).

[13] S. Koskenmies, E. Widén, P. Onkamo, M. Zucchelli, P. Sevon, H. Julkunen, and J. Kere. Haplotype associations define target regions for susceptibility loci in SLE. European Journal of Human Genetics, in press.

[14] M. McPeek and A. Strahs. Assessment of linkage disequilibrium by the decay of haplotype sharing, with application to fine-scale genetic mapping. American Journal of Human Genetics, 65:858-875 (1999).

[15] V. Ollikainen. Simulation techniques for disease gene localization in isolated populations. PhD thesis, University of Helsinki, Department of Computer Science (2002).

[16] P. Onkamo, V. Ollikainen, P. Sevon, H. Toivonen, H. Mannila, and J. Kere. Association analysis for quantitative traits by data mining: QHPM. The Annals of Human Genetics, 66:419-429 (2002).

[17] N. Paunu, P. Lahermo, P. Onkamo, V. Ollikainen, P. Helen, I. Rantala, K. Simola, J Kere, and H. Haapasalo. A novel low-penetrance susceptibility locus for familial glioma at 15q23q26.3. Cancer Research, 62:3798-3802 (2002).

[18] L. Pitt and R. Reinke. Polynomial-time solvability of clustering and conceptual clustering problems: The agglomerative-hierarchical algorithm. Technical Report UIUCDCS-R-871371, University of Illinois, Department of Computer Science (1987).

[19] A. Polvi, T. Polvi, P. Sevon, T. Petäys, T. Haahtela, L. A. Laitinen, J. Kere, and T. Laitinen. Physical map of asthma susceptibility locus in 7p15-p14 and an association study of TCRG. European Journal of Human Genetics, 10:658-665 (2002). 
[20] P. Sevon, V. Ollikainen, P. Onkamo, H. Toivonen, H. Mannila, and J. Kere. Mining associations between genetic markers, phenotypes, and covariates. Genetic Epidemiology, 21(Suppl 1):S588-S593 (2001).

[21] H. Toivonen, P. Onkamo, K. Vasko, V. Ollikainen, P. Sevon, H. Mannila, M. Herr, and J. Kere. Data mining applied to linkage disequilibrium mapping. American Journal of Human Genetics, 67(1):133-145 (2000).

[22] H. Toivonen, P. Onkamo, K. Vasko, V. Ollikainen, P. Sevon, H. Mannila, and J. Kere. Gene mapping by haplotype pattern mining. In IEEE International Symposium on Bio-Informatics \& Biomedical Engineering, pages 99-108 (2000).

[23] S. Zhang and H. Zhao. On a family-based haplotype pattern mining method for linkage disequilibrium mapping. In Proceedings of Pacific Symposium on Biocomputing, pages 100111 (2002). 


\section{Appendix A: Simulation of data}

A simulated data set is used for examples in the chapter. The simulation was carried out using Populus-package by Vesa Ollikainen [15].

The simulated population expands from the initial 100 individuals to 80,000 over 20 generations. Random mating and meioses were simulated at each generation. A single $100 \mathrm{cM}$ chromosome is considered with 101 evenly spaced microsatellite markers. In the initial population there are four alleles at each marker; a single allele with frequency 0.4 , and three alleles with frequency 0.2. Marker mutations were not simulated.

There are two genes in the simulated chromosome with identical effect on the disease risk. The locations of the genes were randomly selected. At both loci, a mutated allele was inserted to six randomly chosen founder chromosomes. Some founder mutations may have disappeared during the course of the generations.

The disease model is based on liability: a person is affected with probability

$$
p=\frac{e^{L}}{1+e^{L}}
$$

Liability $L$ is defined by

$$
L=5 x_{g 1}+5 x_{g 2}+x_{r} \sqrt{3}+C,
$$

where $x_{g 1}$ and $x_{g 2}$ are indicator variables for the presence of the mutated allele at the two loci, and $x_{r}$ is a normal random variable. $C$ is a constant, whose value is adjusted to obtain prevalence of $4 \%$.

From the final population of 80,000 individuals, 200 affected individuals were randomly selected to form the data set. (The parents were used in the inference of their haplotypes.) 


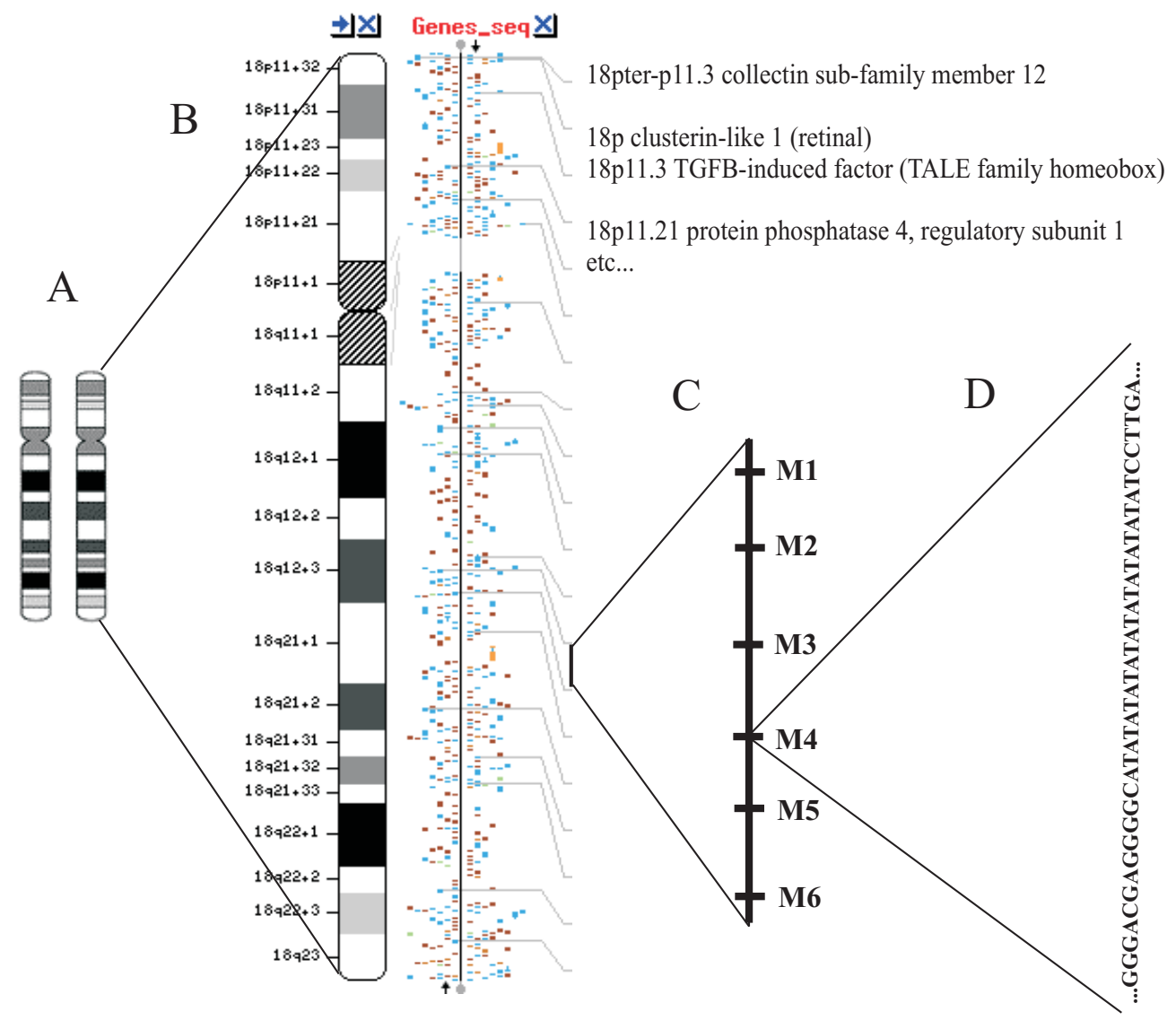

Figure 1: A) A homologous pair of human chromosome 18. B) Enlargement of a chromosome view from NCBI GenBank: the annotated human chromosome 18. Cytogenetic locations are given on the left, and on the right side, the known and predicted genes, shown with colored dots along the vertical line. In the upper right corner there are examples of names of the genes as they appear in NCBI site. C) Zoom-in on a small section of chromosome showing some marker loci (denoted by M1-M6). The alleles at M1-M6 constitute a haplotype. D) Enlargement from C, a stretch of DNA sequence including an 11-repeat allele of locus M4, flanked by unique DNA sequence. 


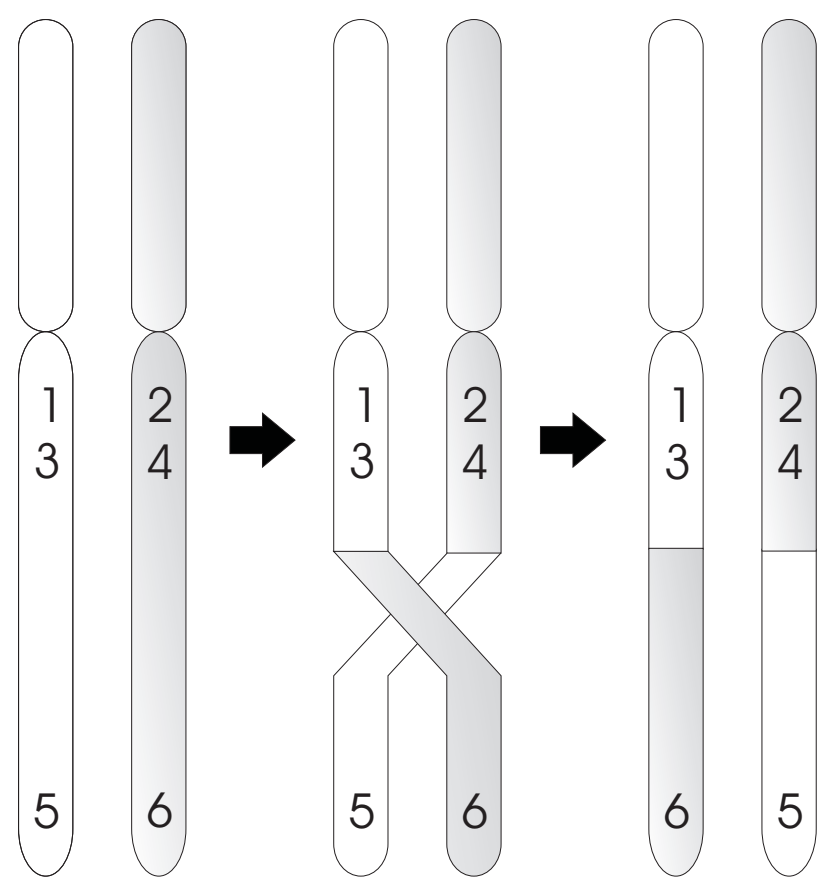

Figure 2: Crossing-over and recombination. Two homologous chromosomes have been duplicated in the process of meiosis. (Here, only two of the four are shown for the sake of simplicity.) Crossing-over and recombination occurs between a pair, chromosomal arms are exchanged (in the middle), and the resulting daughter chromosomes are transmitted to different gametes (in the right). 


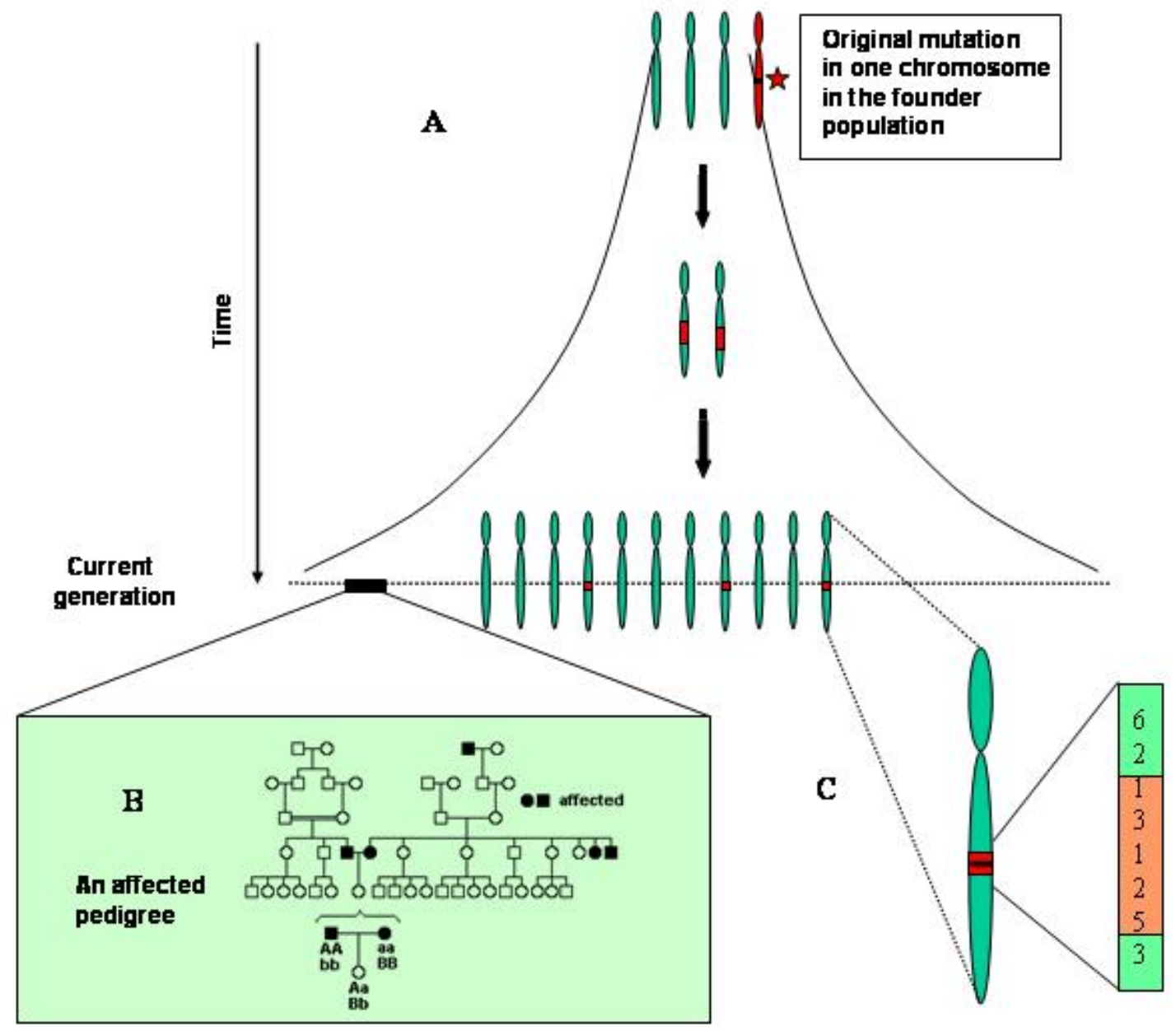

Figure 3: Gene mapping strategies. A) Association analysis. The disease mutation has originated in a common ancestor, and has spread in the population. In the course of generations, consecutive recombinations narrow down the area of conserved haplotype around the disease mutation. In the current generation, only a short stretch of original ancestral haplotype is remaining; now, genotyping a dense map of markers along the chromosome in affected and unaffected individuals and comparing the haplotypes would reveal the area of increased sharing in the disease-associated chromosomes (in red). B) Linkage approach. Co-segregation of the phenotype and genetic markers is tracked in pedigrees of closely related individuals. If several related, affected individuals seem to have inherited the same chromosomal area from a common ancestor more often than based on bare chance, it is deduced that the disease gene is somewhere inside that area. Linkage is often utilized in the first stage of gene mapping project, and when approximate areas of interest have been detected, fine-scale mapping is carried out with association-based methods. C) Enlargement of a disease mutation carrying chromosome. 


\section{Outline of the HPM algorithm}

Input: Marker map, set of disease-associated haplotypes, set of control haplotypes, association threshold.

Output: (Marker, significance) pairs in decreasing order of likelihood of DS gene association.

\section{Method:}

1. Compute a lower bound $l b$ for the frequency of strong patterns

2. Find all patterns that are frequent with respect to $l b$

3. Evaluate the strength of the frequent patterns

4. For each marker $i$, compute the marker frequency $f(i)$ in the strong patterns

5. For $j=1, \ldots, K$ :

6. Randomly permute the status fields of haplotypes

7. Evaluate the strength of the frequent patterns

8. For each marker $i$, compute the marker frequency $f_{j}(i)$ in the strong patterns

9. For each marker $i$ compute $p(i)=\left|\left\{j \mid f_{j}(i) \geq f(i)\right\}\right| / K$

10. Output pairs $(i, p(i))$ sorted by decreasing $p(i)$

Figure 4: The HPM algorithm 

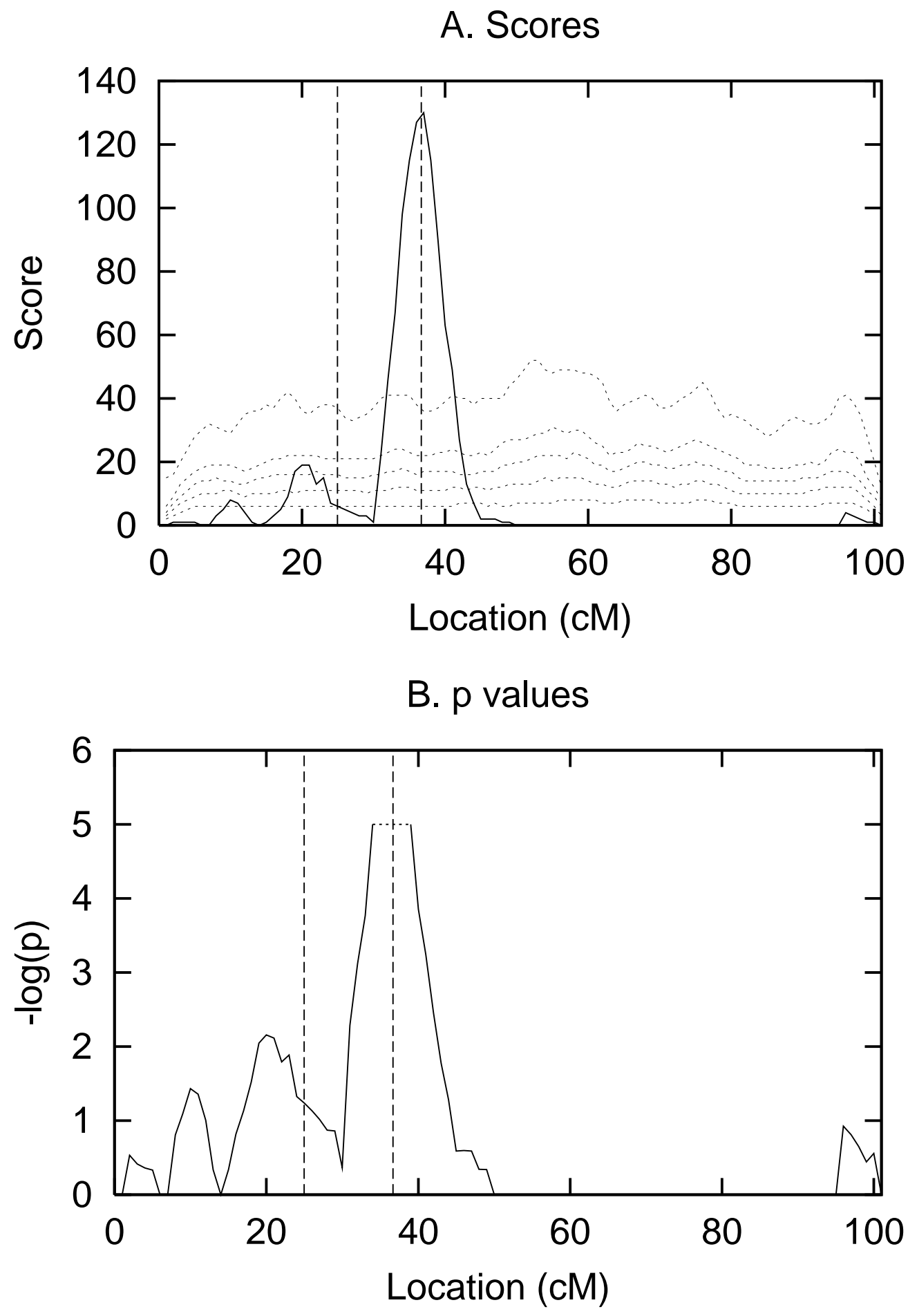

Figure 5: An example of the output of HPM on a simulated data set. A) Observed scores (solid curve) and critical scores for $p$ values $0.001,0.005,0.01,0.02$ and 0.05 obtained by permutation tests (dotted curves). B) Negated logarithms (base 10) of the $p$ values. The finite number of permutations causes a cut-off at $y=5$. The dashed vertical lines denote the true gene loci. 
A. Type 1 diabetes

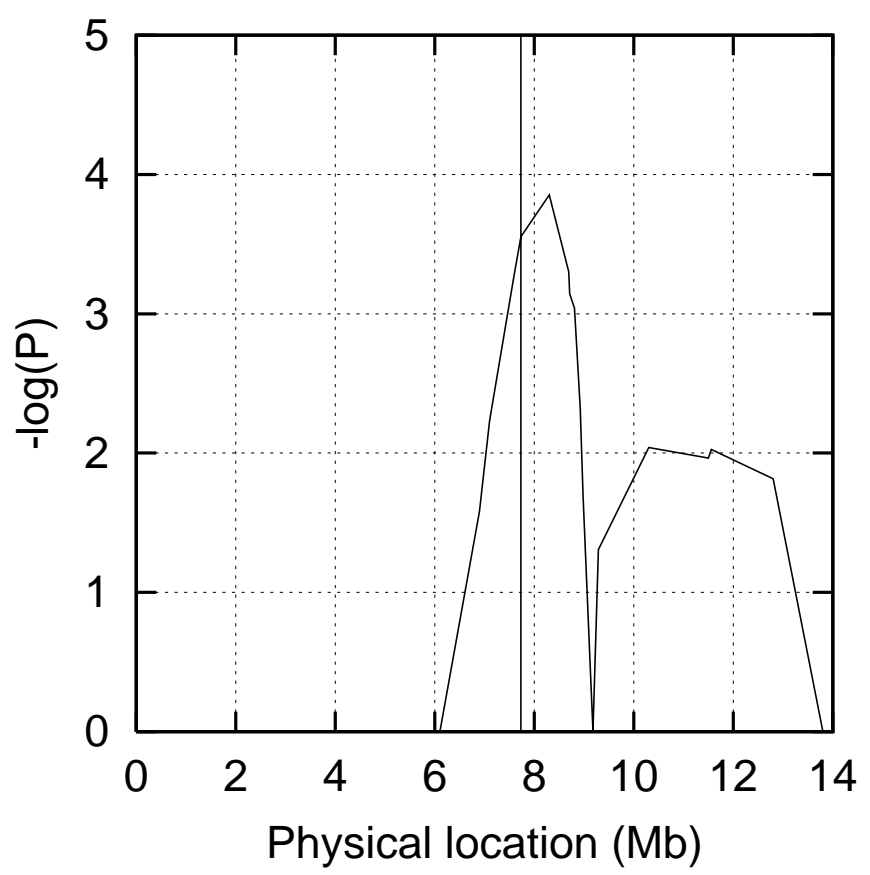

B. SLE

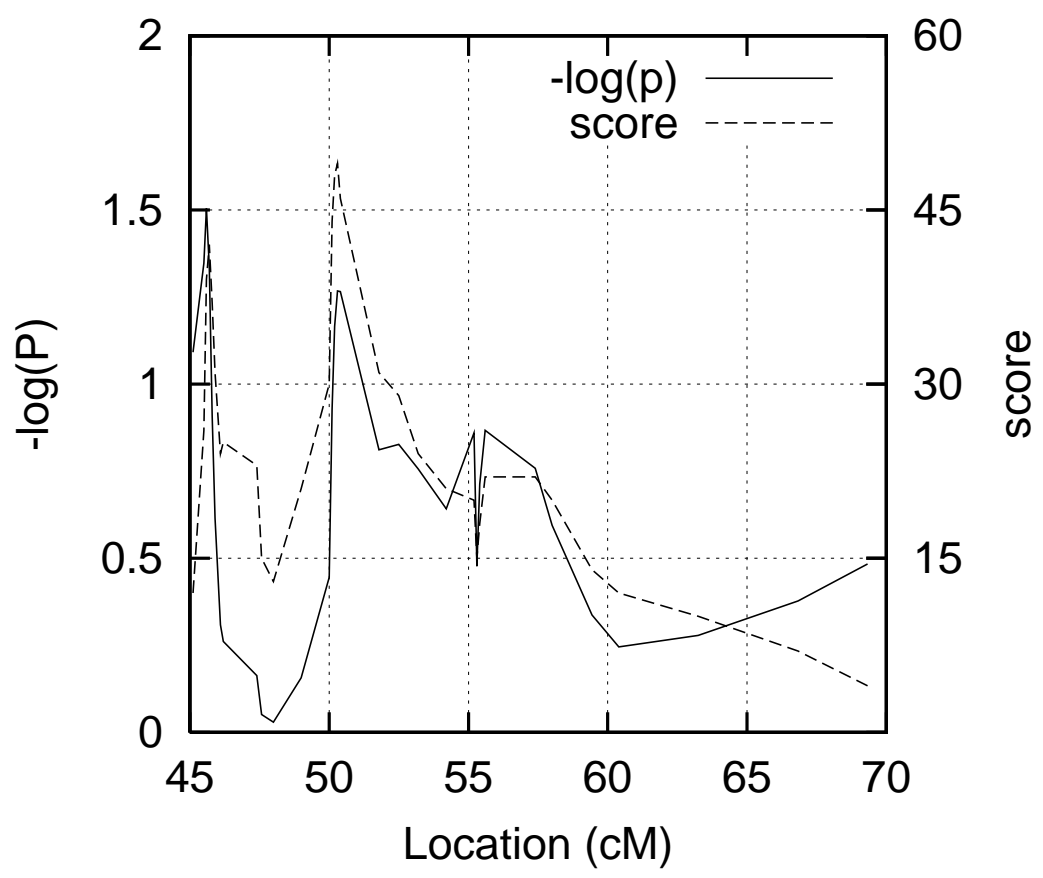

Figure 6: A) Localization of Type 1 diabetes susceptibility genes. The location of the known susceptibility gene is denoted by the solid vertical line. B) Results for the SLE data set. The susceptibility gene is located near or at $50,30 \mathrm{cM}$. 

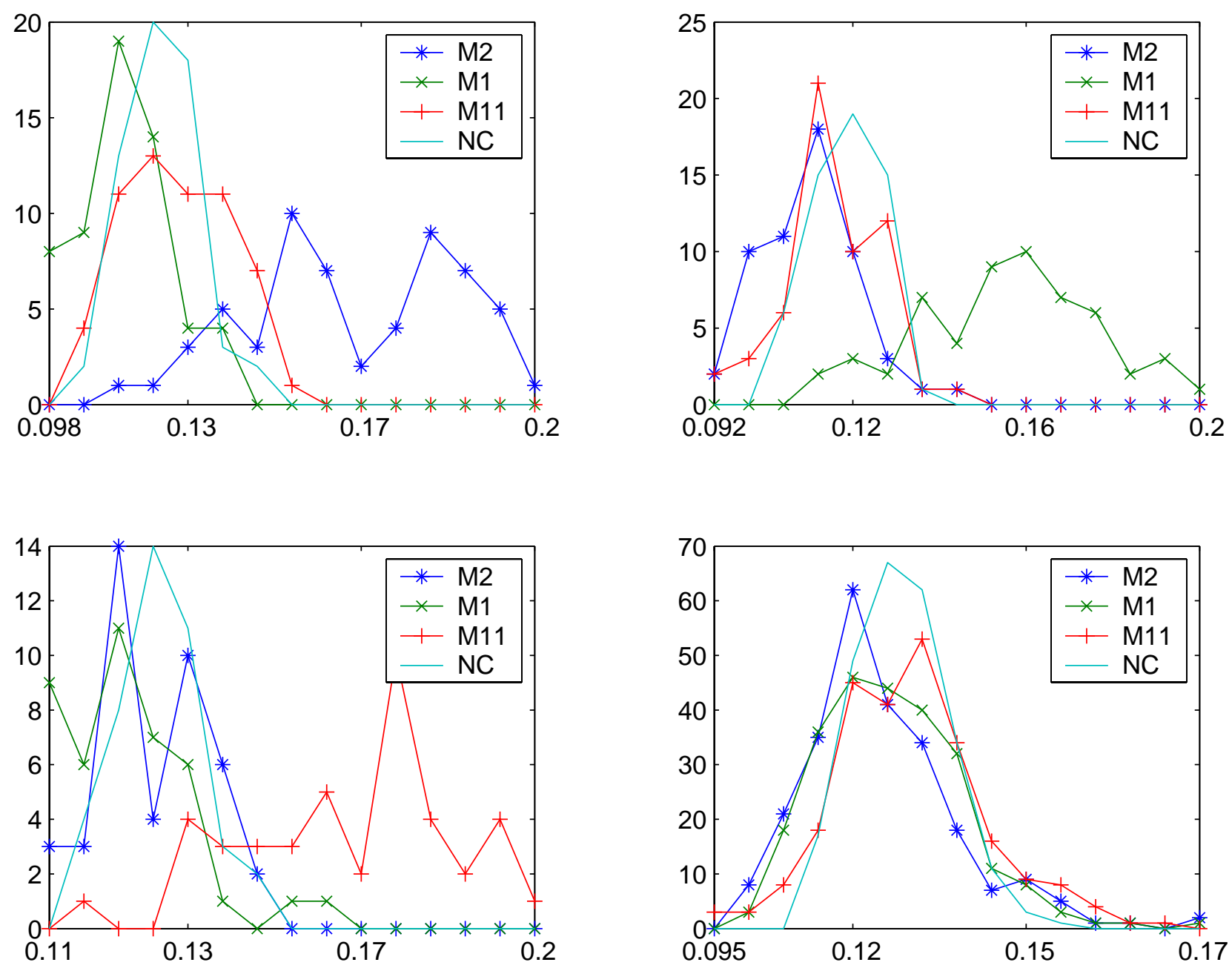

Figure 7: Distributions (histograms) of $S_{H_{i}}$ values in the simulated data. Upper left plot shows the distribution of median similarities of mutation M2 carriers to each of the classes (M1, M2, M11, non-carriers). Other plots contain similar distributions for members of class M1 (top right), M11 (bottom left), and non-carriers (bottom right). Jozef Zurada and Medo Kantardzic (Eds.), IEEE Press 

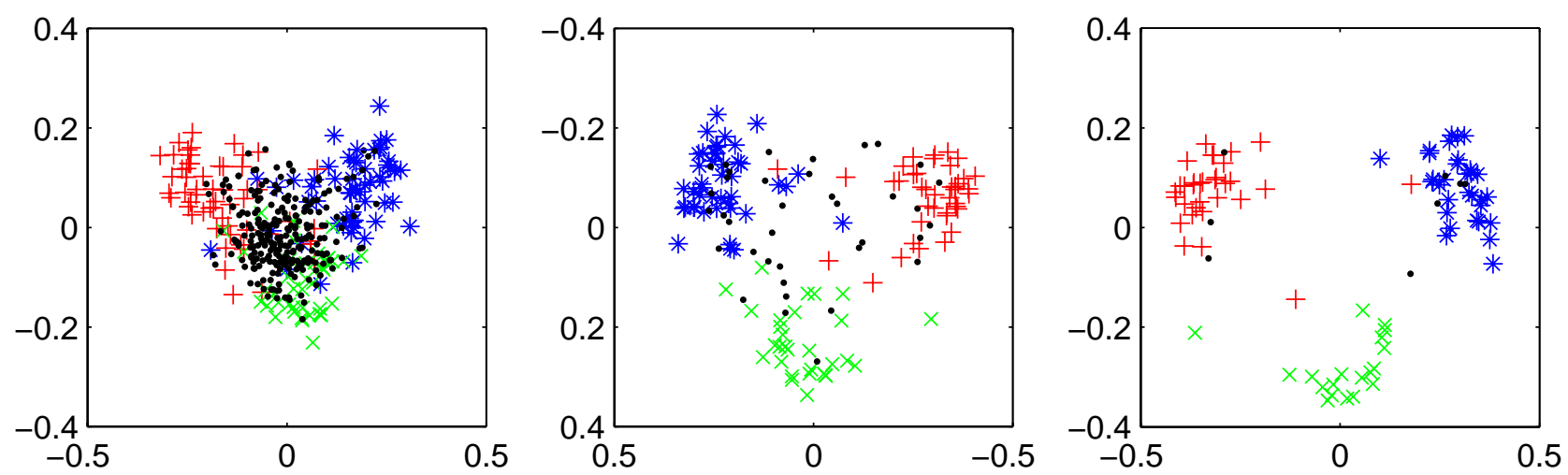

Figure 8: Predicted mutation carriers from the approximative method, with gradually more restricting parameters. Symbols,$+ *$ and $\times$ denote carriers of different mutations; non-carriers are denoted by dots. The plot on the left contains the whole dataset. In the middle plot $(\varepsilon=0.3, t=5)$ most of the carriers are present, but there is visible overlap. In the rightmost plot $(\varepsilon=0.35, t=5)$ the three groups are clearly separate (except for few outliers) from each other and the amount of non-carriers is minimal. 


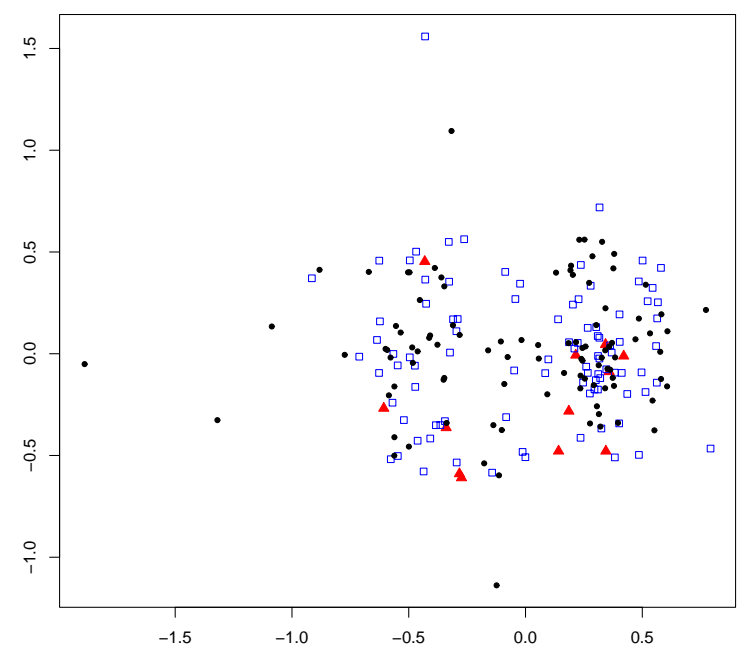

(a)

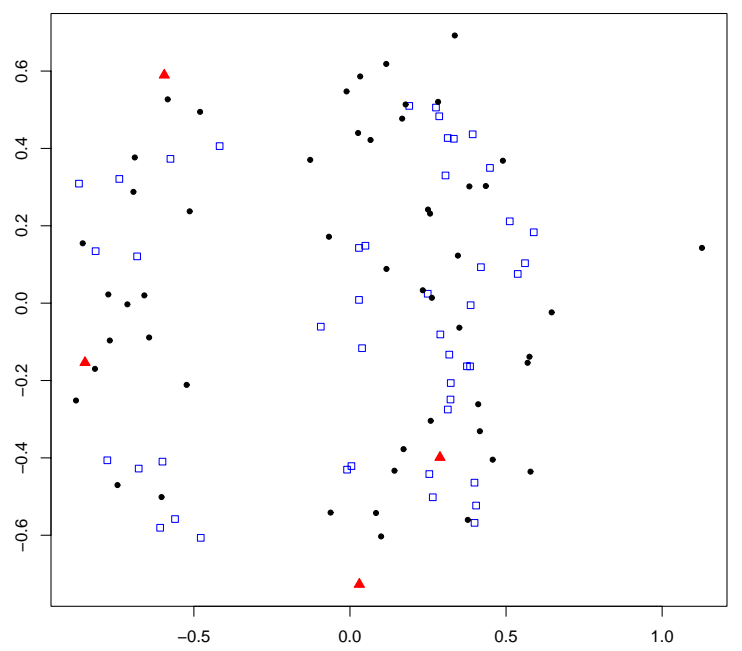

(b)

Figure 9: Experiments with the SLE dataset. Triangles, boxes and dots denote mutation carriers, affected and controls, respectively. A) The whole dataset shows roughly two clusters. B) After applying the approximation heuristic, these two clusters become more obvious. 


\section{Outline of the haplotype clustering algorithm}

Input: Set of haplotypes, threshold $p$ of the number of disjunctions per cluster. Output: Set of clusters, the disjunctive concepts for each cluster.

\section{Method:}

1. For each marker $i$

2. form the haplotype segment containment lattice $L A T T_{i}$

of frequent segments containing $i$

3. Select the lattices that have the largest number of nodes and store them in $S \_L A T T \subseteq L A T T=\left\{L_{A T T}, L_{1} A T T_{2}, \ldots, L A T T_{m}\right\}$

4. For each $L A T T_{i} \in S L A T T$

5. $\quad$ select the $p$ highest scoring nodes of $L A T T_{i}$

6. output the disjunction of the haplotype segments in the selected nodes

Figure 10: The haplotype clustering algorithm 

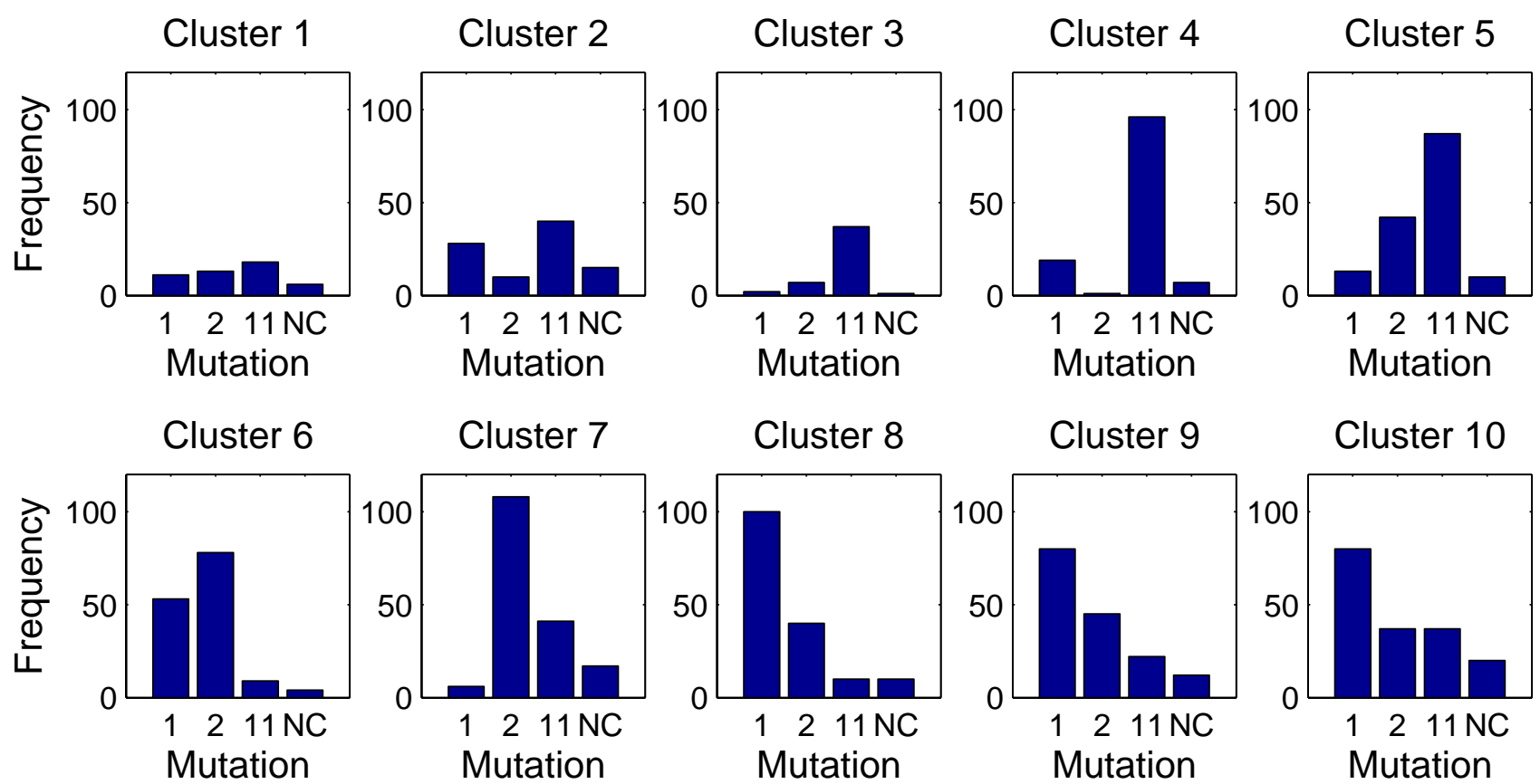

Cluster 10

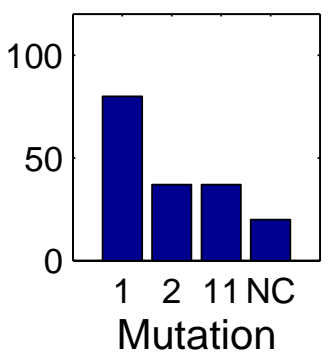

Figure 11: Distributions of carriers of different mutations within different clusters. 


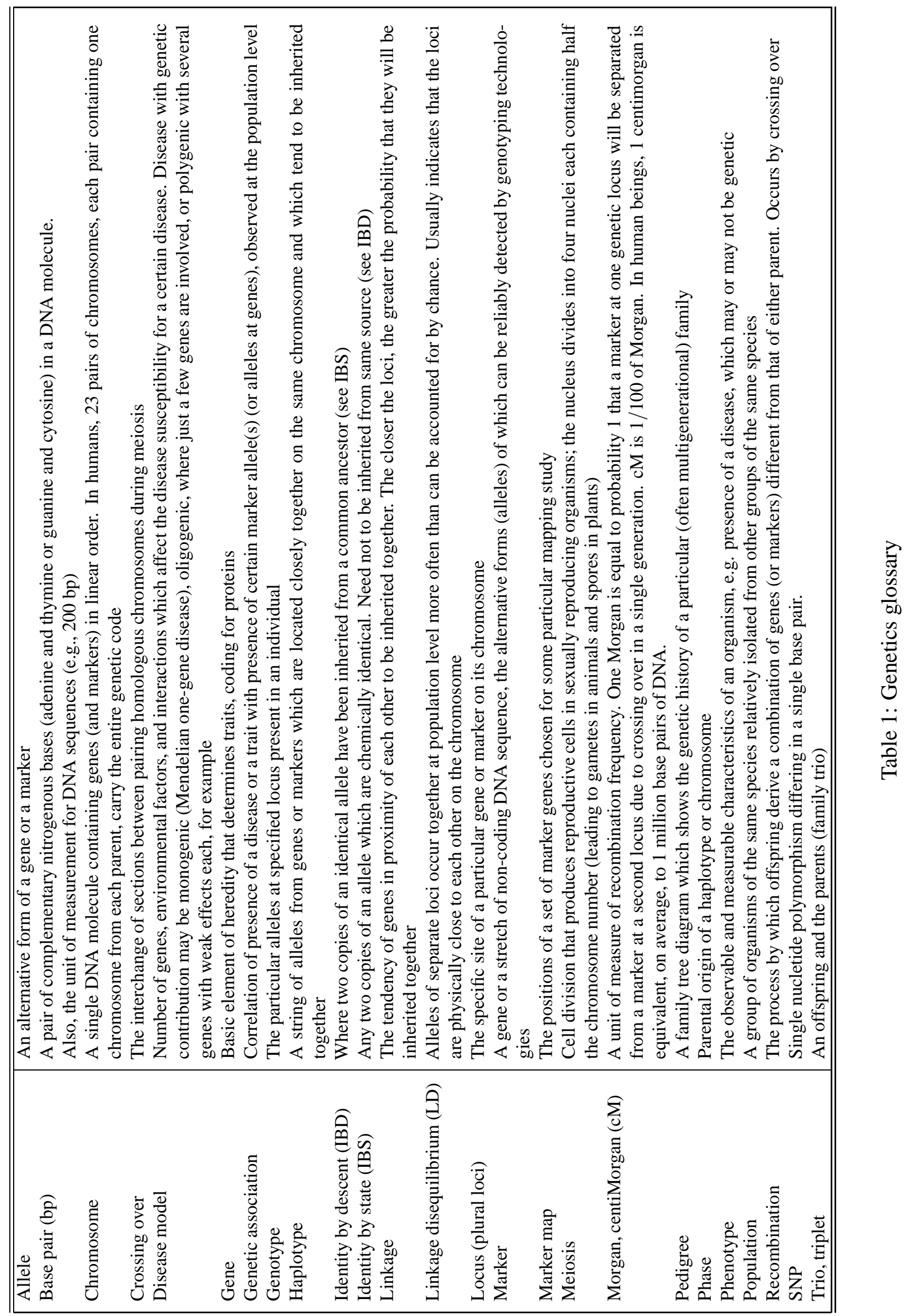




\begin{tabular}{|c|cccccccccc|}
\hline & \multicolumn{10}{|c|}{ Markers/alleles } \\
$\chi^{2}$ & 1 & 2 & 3 & 4 & 5 & 6 & 7 & 8 & 9 & 10 \\
\hline 8.6095 & $*$ & 1 & $*$ & 5 & $*$ & $*$ & $*$ & $*$ & $*$ & $*$ \\
7.1138 & $*$ & $*$ & 5 & $*$ & 6 & $*$ & $*$ & $*$ & $*$ & $*$ \\
6.2421 & $*$ & $*$ & $*$ & $*$ & 6 & $*$ & 1 & 1 & 1 & $*$ \\
5.8522 & $*$ & $*$ & $*$ & $*$ & 6 & $*$ & 2 & 1 & $*$ & $*$ \\
5.8163 & $*$ & $*$ & $*$ & $*$ & $*$ & 3 & 2 & $*$ & 2 & $*$ \\
5.3402 & $*$ & 1 & 1 & $*$ & 6 & 2 & $*$ & $*$ & $*$ & $*$ \\
5.3402 & $*$ & 1 & 1 & $*$ & 6 & $*$ & $*$ & $*$ & $*$ & $*$ \\
5.2571 & $*$ & $*$ & 1 & $*$ & 6 & $*$ & $*$ & $*$ & $*$ & $*$ \\
5.0892 & $*$ & $*$ & $*$ & $*$ & 6 & $*$ & 1 & 1 & $*$ & $*$ \\
\hline
\end{tabular}

Table 2: Haplotype patterns over markers 1-10 strongly associated with SLE

\begin{tabular}{|l|l|l|}
\hline Class & Haplotypes in total & Predicted mutation carriers \\
\hline M2 carriers & $58(14.5 \%)$ & $32(35.2 \%)$ \\
M1 carriers & $56(14.0 \%)$ & $32(35.2 \%)$ \\
M11 carriers & $42(10.5 \%)$ & $19(20.9 \%)$ \\
Non-carriers & $244(61.0 \%)$ & $8(8.7 \%)$ \\
\hline Total & 400 & 91 \\
\hline
\end{tabular}

Table 3: Number of predicted mutation carriers (approximative method)

\begin{tabular}{|l|ccccccccc|}
\hline & \multicolumn{10}{|c|}{ Markers/alleles } \\
& 1 & 2 & 3 & 4 & 5 & 6 & 7 & 8 & 9 \\
\hline haplotype $H_{1}$ & 1 & 4 & 1 & 2 & 1 & 2 & 3 & 4 & 3 \\
haplotype $H_{2}$ & 3 & 1 & 4 & 4 & 4 & 4 & 3 & 4 & 3 \\
haplotype $H_{3}$ & 1 & 3 & 1 & 1 & 1 & 3 & 3 & 3 & 3 \\
\hline
\end{tabular}

Table 4: Example of three haplotypes and their similarities

\begin{tabular}{|l|lllllll|}
\hline & \multicolumn{7}{|c|}{ Markers/alleles } \\
& 85 & 86 & 87 & 88 & 89 & 90 & 91 \\
\hline $85:$ & 4 & 3 & 1 & 3 & - & - & - \\
$88:$ & - & - & - & 3 & 4 & 2 & 3 \\
$85:$ & 3 & 3 & 2 & 3 & - & - & - \\
$88:$ & - & - & - & 3 & 2 & 1 & 4 \\
\hline
\end{tabular}

Table 5: Example of a disjunctive concept 


\begin{tabular}{|c|c|c|c|c|c|c|c|c|}
\hline Cluster 1 & $\mathrm{C}$ & $\mathrm{NC}$ & Cluster 5 & $\mathrm{C}$ & $\mathrm{NC}$ & Cluster 9 & $\mathrm{C}$ & $\mathrm{NC}$ \\
\hline $15:[1]$ & 10 & 34 & $9:[1]$ & 7 & 52 & $6:[2]$ & 5 & 33 \\
\hline $15:[1,3]$ & 6 & 10 & $9:[1,2]$ & 1 & 14 & $6:[2,2]$ & 1 & 10 \\
\hline $15:[1,1]$ & 3 & 16 & $9:[1,11]$ & 2 & 2 & $6:[2,1]$ & 2 & 17 \\
\hline $15:[1,3,3]$ & 1 & 5 & $9:[1,5]$ & 2 & 10 & $6:[2,2,5]$ & 0 & 5 \\
\hline $14:[3,1,1]$ & 3 & 1 & $8:[5,1,1]$ & 0 & 3 & $5:[6,2,2,5]$ & 0 & 3 \\
\hline Cluster 2 & $\mathrm{C}$ & $\mathrm{NC}$ & Cluster 6 & $\mathrm{C}$ & $\mathrm{NC}$ & Cluster 10 & $\mathrm{C}$ & $\mathrm{NC}$ \\
\hline $19:[1]$ & 8 & 44 & \begin{tabular}{|l|}
$11:[1]$ \\
\end{tabular} & 7 & 52 & $30:[1]$ & 5 & 56 \\
\hline $19:[1,1]$ & 1 & 12 & $11:[1,1]$ & 0 & 12 & $29:[11,1]$ & 0 & 6 \\
\hline $19:[1,3]$ & 3 & 7 & $10:[2,1,1]$ & 0 & 5 & $30:[1,2,3]$ & 1 & 6 \\
\hline $18:[1,1,3]$ & 1 & 5 & $11:[1,1,4]$ & 0 & 9 & $29:[7,1,1]$ & 0 & 4 \\
\hline $18:[1,1,3,2]$ & 1 & 4 & $10:[2,1,1,4]$ & 0 & 5 & $29:[7,1,1,3]$ & 0 & 4 \\
\hline Cluster 3 & $\mathrm{C}$ & $\mathrm{NC}$ & Cluster 7 & $\mathrm{C}$ & $\mathrm{NC}$ & Cluster 11 & C & $\mathrm{NC}$ \\
\hline $21:[2]$ & 8 & 68 & \begin{tabular}{|l|}
$17:[3]$ \\
\end{tabular} & 6 & 50 & $13:[4]$ & 1 & 44 \\
\hline $21:[2,1]$ & 0 & 13 & $17:[3,1]$ & 1 & 26 & $13:[4,2]$ & 0 & 21 \\
\hline $21:[2,8]$ & 5 & 15 & $16:[3,3,1]$ & 0 & 12 & $13:[4,1]$ & 0 & 15 \\
\hline $20:[3,2,8]$ & 2 & 5 & $16:[4,3,1]$ & 0 & 4 & $12:[1,4,1]$ & 0 & 5 \\
\hline $19:[1,3,2,8]$ & 2 & 3 & $17:[3,1,1,3]$ & 1 & 4 & $12:[4,4,2]$ & 0 & 3 \\
\hline Cluster 4 & $\mathrm{C}$ & $\mathrm{NC}$ & Cluster 8 & $\mathrm{C}$ & $\mathrm{NC}$ & & & \\
\hline $2:[1]$ & 7 & 56 & $27:[1]$ & 6 & 43 & & & \\
\hline $1:[4,1]$ & 1 & 10 & $27:[1,1]$ & 1 & 7 & & & \\
\hline $2:[1,1]$ & 2 & 11 & $26:[3,1,1]$ & 0 & 4 & & & \\
\hline $2:[1,1,3]$ & 2 & 6 & $25:[3,3,1,1]$ & 0 & 3 & & & \\
\hline $2:[1,1,3,6]$ & 2 & 4 & $25:[3,3,1,1,7]$ & 0 & 3 & & & \\
\hline
\end{tabular}

Table 6: Cluster descriptions for the affected individuals in the SLE data. In all cases, the first line in the description is the only logically non-redundant disjunct, but others are listed for their potential information to the user. The distributions of carriers (C) and non-carriers (NC) of the mutation are also shown for each disjunct. 